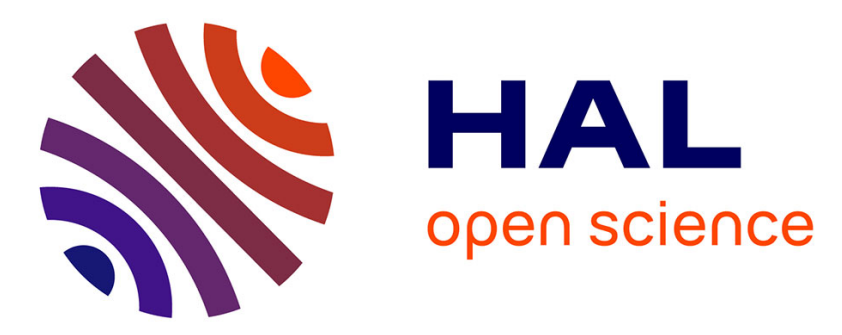

\title{
Mettre les polythéismes en formules? À propos de la base de données Mapping Ancient Polytheisms
}

\author{
Sylvain Lebreton, Corinne Bonnet
}

\section{To cite this version:}

Sylvain Lebreton, Corinne Bonnet. Mettre les polythéismes en formules? À propos de la base de données Mapping Ancient Polytheisms. Kernos - Revue internationale et pluridisciplinaire de religion grecque antique, 2019, 32, pp.267-296. 10.4000/kernos.3163 . hal-02555965

\section{HAL Id: hal-02555965 \\ https://hal.science/hal-02555965}

Submitted on 27 Apr 2020

HAL is a multi-disciplinary open access archive for the deposit and dissemination of scientific research documents, whether they are published or not. The documents may come from teaching and research institutions in France or abroad, or from public or private research centers.
L'archive ouverte pluridisciplinaire HAL, est destinée au dépôt et à la diffusion de documents scientifiques de niveau recherche, publiés ou non, émanant des établissements d'enseignement et de recherche français ou étrangers, des laboratoires publics ou privés. 


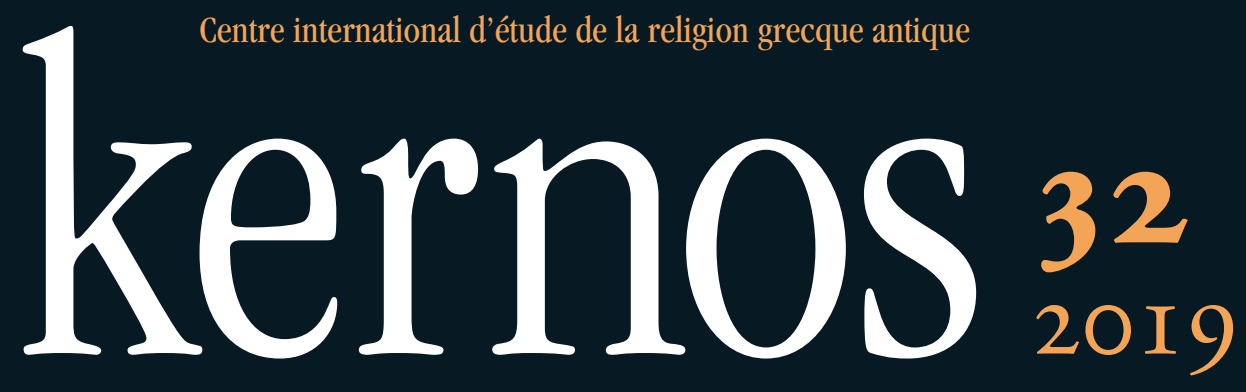

Revue internationale et pluridisciplinaire de religion grecque antique

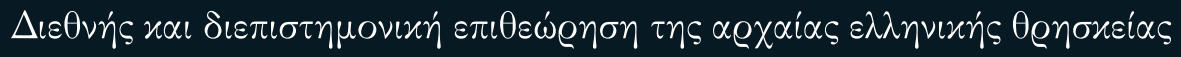

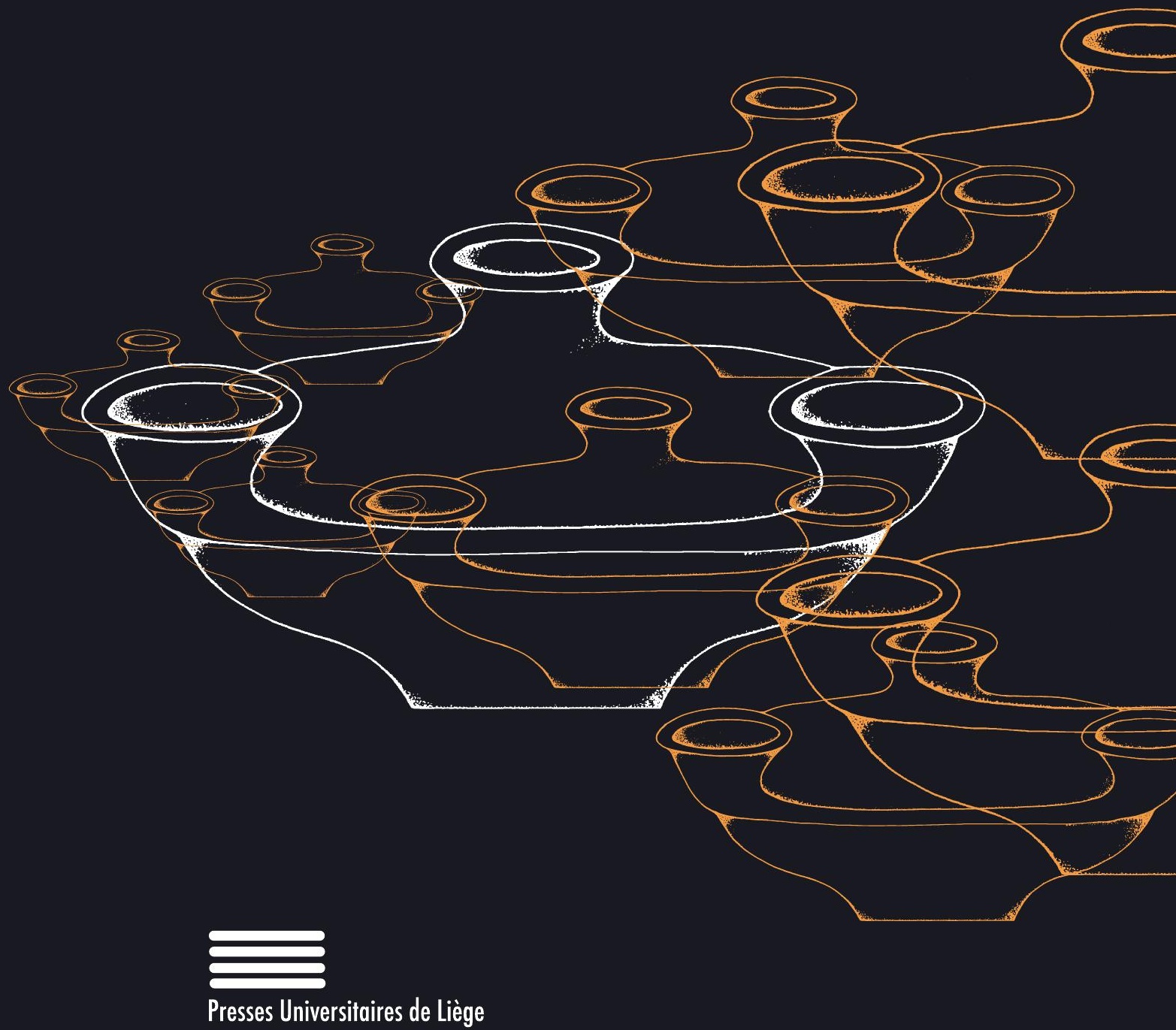




\section{Comité de rédaction}

André Motte (Université de Liège), président du comité de rédaction; Vinciane Pirenne-Delforge (Collège de France - Université de Liège), directrice de la revue ; Jan-Mathieu Carbon (Collège de France - Université de Liège), secrétaire scientifique; Pierre Brulé (Université de Rennes 2); Claude Calame (École Pratique des Hautes Études en Sciences Sociales, Paris); Angelos Chaniotis (Institute for Advanced Study, Princeton); Emilio Suárez de la Torre (Université Pompeu Fabra de Barcelone); Didier Viviers (Université libre de Bruxelles); Emmanuel Voutiras (Université de Thessalonique).

\section{Comité consultatif}

Pierre Bonnechere (Montréal); Corinne Bonnet (Toulouse); Philippe Borgeaud (Genève); Stefano Caneva (Liège); Véronique Dasen (Fribourg); Gunnel Ekroth (Uppsala); Pierre Ellinger (Paris); Stella Georgoudi (Paris); Anne-Françoise Jaccottet (Genève); Dominique Jaillard (Genève); Sarah Iles Johnston (Ohio); Madeleine Jost (Paris); Joannis Mylonopoulos (New York); Massimo Osanna (Naples); Gabriella Pironti (Paris); François de Polignac (Paris); Eftychia Stavrianopoulou (Heidelberg); Annie VerbanckPiérard (Mariemont).

\section{Courrier scientifique}

Les manuscrits (voir les instructions sur le site internet de la revue, http://www.kernos.ulg.ac.be), les livres et les numéros de revue envoyés pour recension sont à adresser à :

Revue Kernos, Université de Liège, 7, place du 20-Août, 4000 Liège (Belgique). Tél. +32 43665568 / adresse courriel : kernos@ulg.ac.be

\section{Diffusion et vente \\ Editions De Boccard}

4, rue de Lanneau

75006 Paris (France)

Tél. +33143260037 / Fax +331435485 83 / http://www.deboccard.com Toutes les commandes ou demandes d'information doivent être adressées : - pour l'abonnement à la revue Kernos ou l'achat au numéro, à orders@deboccard.com

- pour l'acquisition des suppléments, à info@deboccard.com 


\section{Table des matières}

Éditorial, par André MotTe et Vinciane Pirenne-Delforge

Hommage à Marcel Detienne, par Philippe BORGEAUD

\section{Études}

Bartek BeDnAREK, Orpheus in Aeschylus and the Thracian child-eater on a bydria from the British Museum

Claudio BiagetTr, Una menzione di Atena Archegetis in P.Hib. I 15.

Note sull'epiteto e sul suo impiego ad Atene

Sonia Darthou, L'olivier, identité et rempart d'Athènes : un épisème de la cité?

Julie Balériaux, Mythical and ritual landscapes of Poseidon Hippios in Arcadia

Hedvig von Ehrenheim, Causal explanation of disease in the iamata of Epidauros

Dennis D. Hughes, The Cult of Aratus at Sicyon (Plutarch, Aratus, 53)

Stefano Caneva, V ariations dans le paysage sacré de Pergame:

l'Asklépieion et le temple de la terrasse du théâtre

Alaya Palamidis, Des souris et des hommes. Une réinvention érudite du culte d'Apollon Smintheus à l'époque hellénistique?

Denis Rousset, Géographie, paléographie et philologie. Note sur un lieu de culte de Déméter

Anna Angelini, Naming the gods of the others in the Septuagint: lexical analysis and historical-religious implications

Corinne Bonnet, Sylvain LeBreton, Mettre les polythéismes en formules? À propos de la Base de Données Mapping Ancient Polytheisms

\section{Chronique des activités scientifiques}

Epigraphic Bulletin 2016, by Angelos CHANiOTIS

Revue des Livres 


\section{Comptes rendus et notices bibliographiques}

C. Semenzato, À l'écoute des Muses en Grèce archaïque (A. Motte) ...............................................341

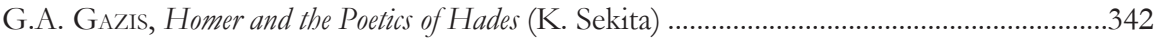

C. SÁNCHEZ MAÑas, Los oráculos en Heródoto (M. Giangiulio).......................................................344

G.H. Renberg, Where Dreams May Come (R. Van Hove) ..........................................................347

F. Pasche Guignard et al. (éd.), Maternità e politeismi (Fr. Prescendi) .....................................350

A. Konstantinou, Female Mobility and Gendered Space in Ancient Greek. Myth (M. Augier) ..352

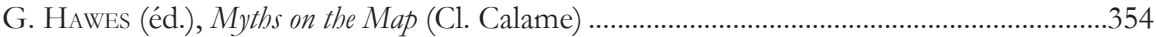

S.I. Johnston, The Story of Myth (V. Pirenne-Delforge) ............................................................358

Y. Ustinova, Divine Mania (M. Lesgourgues) …………………………………………........361

A. Rolle, Dall'Oriente a Roma. Cibele, Iside e Serapide nell'opera di V arrone (L. Beaurin) .........364

A. LujJENDIJK, W.E. KuIngshirn (éd.), My Lots are in Thy Hands (F. Spadini).......................366

2. Actes de colloques, ouvrages collectifs et anthologies ...........369

3. Ouvrages reçus à la rédaction ..................................376

Revue des Revues, par Hélène CoLlard et Zoé PitZ ..................................379 
Kernos 32 (2019), p. 267-296.

\section{Mettre les polythéismes en formules? À propos de la base de données Mapping Ancient Polytbeisms}

Résumé : Cet article présente la Base de Données (BDD) développée dans le cadre du projet ERC Mapping Ancient Polytheisms (MAP), qui vise à étudier les systèmes religieux des mondes grecs et ouest-sémitiques dans la longue durée (ca. 1000 av. - 400 ap. J.-C.) à travers le prisme des «attributs onomastiques divins ». On entend par là les noms, épithètes, formes verbales, et autres appellations que les Anciens attribuaient à leurs dieux. La construction d'un tel outil, qui n'est pas sans précédents mais qui s'efforce de franchir un seuil quantitatif et qualitatif, répond à un double impératif: permettre le traitement exhaustif des myriades de dénominations divines en prenant en compte la diversité de contextes dans lesquels elles apparaissent; utiliser ces dénominations comme les révélateurs des configurations divines et de l'agentivité humaine. La complexité inhérente aux séquences onomastiques divines nécessite l'élaboration d'une base de données soigneusement structurée, souple et précise à la fois. Ainsi, à la suite d'une mise au point terminologique et historiographique, on présente ici la structure de la BDD MAP, et notamment le dispositif d'analyse des formules qui permet la modélisation des séquences onomastiques divines.

\footnotetext{
Abstract: This paper presents the Database (DB) developed within the ERC Mapping Ancient Polytheisms (MAP) project, which aims to study the religious systems of the Greek and West Semitic worlds in the longue durée (ca. $1000 \mathrm{BCE}-400 \mathrm{CE}$ ) through the prism of "divine onomastic attributes". By this we mean the names, epithets, verbal forms, and other designations that the Ancients attributed to their gods. The construction of such a tool, which is not without precedent, but which strives to take a quantitative and qualitative step forward, has a double objective: on the one hand, to allow the exhaustive treatment of myriads of divine denominations, by taking into account the diversity of contexts in which they appear; on the other, to use these denominations as markers of divine configurations and human agency. The complexity inherent in divine onomastic sequences requires the development of a carefully structured, flexible and precise database. Therefore, an exhaustive treatment of the latter requires the development of flexible modelling. Thus, after a terminological and historiographical discussion, we here present the structure of the MAP database, and in particular the formula analysis that allows the modelling of divine onomastic sequences.
} 


\section{1. ÉPITHÈTES, ÉPICLÈSES ET ATTRIBUTS ONOMASTIQUES DIVINS}

\subsection{Nommer les dieux : les enjeux d'un projet comparatiste ${ }^{1}$}

En ciblant les modes de dénomination des dieux, le projet « Mapping Ancient Polytheisms » s'attache à explorer les processus de définition, d'organisation et de fonctionnement des mondes divins dans une vaste Antiquité méditerranéenne ${ }^{2}$. On présente ici la base de données (BDD) qui constitue l'axe central du projet ${ }^{3}$. Sa construction n'est en effet pas un acte purement technique, mais engage au contraire les fondements mêmes de la démarche et impose une réflexion approfondie sur les présupposés méthodologiques et les catégories les plus aptes à capturer et rendre compte de l'extrême complexité des systèmes religieux étudiés. Un premier article collectif a été publié fin 2018 dans la revue Studi e materiali di storia delle religioni ${ }^{4}$, afin d'exposer les premières propositions du projet MAP en matière de catégories et de terminologie. Celles-ci dérivent directement des efforts accomplis pour définir des « ontologies » pertinentes et efficaces permettant d'offrir, à travers la BDD, une représentation aussi claire que possible des systèmes religieux et de leur fonctionnement 5 .

En partant du principe que la manière dont les entités surhumaines sont désignées est significative de leurs natures, fonctions, modes opératoires et de leurs agencements au sein d'ensembles, le projet MAP vise à répertorier et traiter systématiquement l'ensemble des données disponibles dans deux aires géoculturelles intrinsèquement méditerranéennes et fortement connectées : le

1. Sauf indication contraire, les dates sont entendues avant notre ère.

2. «Mapping Ancient Polytheisms. Cult Epithets as an Interface between Religious Systems and Human Agency » (MAP) est un projet ERC Advanced Grant dirigé par Corinne Bonnet, université Toulouse - Jean Jaurès, laboratoire PLH-ERASME, pour une durée de cinq ans (oct. 2017 - oct. 2022). L'équipe est actuellement composée d'une coordinatrice de projet, Élodie Guillon, de quatre post-doctorants, Thomas Galoppin, Aleksandra Kubiak-Schneider, Sylvain Lebreton et Fabio Porzia, d'un ingénieur de recherche, Antoine Laurent, et d'une doctorante, Giuseppina Marano; Maria Bianco a été post-doctorante durant les deux premières années du projet. Il est financé par le Conseil Européen de la Recherche (ERC) dans le cadre du programme de recherche et d'innovation de l'Union Européenne Horizon 2020 (contrat de financement $\mathrm{n}^{\circ}$ 741182). Nos remerciements vont à Vinciane Pirenne-Delforge qui nous a conviés à présenter la base de données MAP dans Kernos.

3. Étant entendu que les activités du projet ne se limitent pas à la construction de la BDD. Cf. notre site pour le suivi de nos activités : https://map-polytheisms.huma-num.fr/.

4. Bonnet - Bianco - Galoppin - Guillon - Laurent - Lebreton - Porzia (2018), désormais BONNET et al. (2018).

5. Par «ontologie », on entend l'ensemble structuré de termes et concepts qui représentent le sens d'un champ d'informations et qui permettent de spécifier dans un langage formel les concepts d'un domaine et leurs relations. On peut dire que l'ontologie est aux données ce que la grammaire est au langage. Cf. Ide - Woolner (2007); Adorni - Maratea - Pandolfo Pulina (2015). 
domaine grec dans toute son extension et celui que couvrent les langues nord-ouest sémitiques (phénicien et punique, hébreu et araméen), en d’autres termes le Levant. C'est ainsi un ample espace-temps méditerranéen qui est visé, riche en échanges et interactions ${ }^{6}$, en similitudes et écarts, dans la longue durée qui s'étend de 1000 av. n. è. à 400 de n. è. environ, soit un millénaire et demi. Les importantes différences structurelles entre les deux systèmes linguistiques envisagés ont précisément requis une grande souplesse dans les catégories mobilisées pour enregistrer les données dans une seule et même BDD, ce qui s'imposait afin de favoriser les analyses comparatives et de traiter ensemble les textes bilingues (ou trilingues). Le choix de ces deux terrains d'enquête rend in fine possible un questionnement critique des catégories de " polythéisme » et « monothéisme » par la comparaison des pratiques onomastiques.

Compte tenu de la richesse de la documentation disponible, des priorités ont dû être définies : l'enjeu de la constitution d'une BDD est en effet de fournir aux usagers des ensembles complets, donc des résultats fiables et exploitables. Par conséquent, si la BDD est conçue pour enregistrer tout type de source écrite, ce sont d'abord les inscriptions qui sont traitées, l'objectif majeur du projet MAP étant de mettre à la disposition de la communauté, fin 2022, un outil aussi exhaustif que possible dans ce domaine. Hébergée par la TGIR Huma-Num ${ }^{7}$, la BDD sera disponible en open access via le site internet du projet ${ }^{8}$. Nous espérons l'ouvrir au public fin 2020 lorsqu'un nombre significatif de corpus épigraphiques aura été entièrement enregistré. Dans cette optique, les premiers efforts portent sur des régions riches en inscriptions tant sémitiques que grecques (Chypre, Phénicie, Carthage).

Parallèlement aux inscriptions, ce sont également les papyrus, monnaies et gemmes que l'équipe de MAP scrute à la recherche de noms attribués aux dieux. Quant aux sources issues de la tradition manuscrite (les sources dites « littéraires ») autre dossier tentaculaire, elles sont pour l'heure intégrées dans des analyses qualitatives et pourront faire l'objet de sondages à partir de tel ou tel auteur pour la BDD. L'après-MAP, que l'on imagine a priori comme un projet international et

6. Sur ces questions, voir notamment PARKER (2017a).

7. Très Grande Infrastructure de Recherche, URL : https://www.huma-num.fr/ (07/18).

8. URL : https://map-polytheisms.huma-num.fr/ (10/18).

9. Il faut ajouter que la dichotomie « inscriptions » / « sources littéraires » présente des limites et soulève des problèmes : un hymne gravé sur le mur d'un sanctuaire, par exemple, relève des deux catégories; quant à l'Ancien Testament, il rassemble une collection de textes de nature très variée, certains très "littéraires ", d'autres " historiques ", d'autres encore "normatifs " ou "prescriptifs ", très similaires à ce que les normes rituelles grecques renferment comme informations, sans oublier les textes « liturgiques » que sont les Psaumes, saturés d'invocations au dieu unique désigné sous une myriade d'appellations, d'un grand intérêt pour le projet MAP. 
collaboratif, pourra prendre le relais sur leur traitement systématique et sur la prise en compte d'autres aires géo-culturelles dans la $\mathrm{BDD}^{10}$.

Or, dès lors que le travail de conception de la BDD a commencé, l'incroyable variété des documents et, partant, des façons de nommer les dieux, nous a, en quelque sorte, sauté aux yeux. L'enjeu même du projet MAP est de rendre compte de cette variété en tant que, d'une part, mode de connaissance et de structuration du divin et, d'autre part, outil empirique pour interagir aussi efficacement que possible avec les dieux. Les notions de « théonyme » et d' " épithète » ou " épiclèse » utilisées jusque-là de manière assez systématique pour décrire les appellations composites désignant les dieux nous sont vite apparues comme trop rigides et trop schématiques. Face à la diversité et la complexité des données contenues dans les sources, nous avons donc proposé, dans l'article mentionné ci-dessus, de parler de séquence ou formule onomastique ${ }^{11}$, chaque élément de celle-ci étant un attribut onomastique. Les dieux sont certes souvent dénommés au moyen d'un binôme comprenant un nom propre et une qualification (adjectif ou substantif), comme Athéna Polias, "Athéna de la polis» ou Reshef has ("Reshef de la flèche »), mais d'autres combinaisons ne sont pas rares, qui peuvent prendre la forme de phrases nominales ou verbales, juxtaposer plusieurs noms propres, inclure des toponymes, etc. Par ailleurs, la notion de « théonyme » suppose d'identifier une tête de syntagme à laquelle on attribue une fonction dominante, ce qui est parfois impossible : en effet, les éléments peuvent être juxtaposés sans qu'une hiérarchisation ne s’impose vraiment, comme dans le cas de Zeus Hēlios Megas Sarapis ${ }^{12}$.

La grande variété de composition des séquences onomastiques divines est particulièrement patente dans la démarche visant à qualifier une divinité en lien avec l'espace, qui constitue la pratique de loin la mieux représentée, sans être pour autant, en aucune façon, banale ${ }^{13}$. Pour convoquer un aspect particulier d'un dieu ou d'une déesse, on a fréquemment recours à une qualification en lien avec l'espace,

10. Elle est en effet conçue, s'agissant d'un outil en open access, pour accueillir d'autres projets portant sur des objets analogues. Un premier transfert est en cours d'étude vers le projet EPIDI de l'université de Madrid Carlos III, sur les noms divins dans l'épigraphie latine d'Hispanie, dirigé par J. Alvar (https://xligirea.weebly.com/jaime-alvar.html). La réflexion sur d'autres extensions est en cours.

11. Nous renvoyons à notre formulation de BONNET et al. (2018), p. 568, n. $3:$ : La notion de séquence permet d'appréhender un ensemble d'éléments onomastiques sans présumer de leur nombre, ordre d'apparition, etc.; la notion de formule, empruntée au domaine des mathématiques ou de la chimie, souligne le fait que chaque élément possède des propriétés particulières et que leur association ou liaison, selon des modalités variées, donne naissance à un "produit » qui n'est pas simplement la somme de ses composés. En chimie comme en mathématiques, les formules constituent une écriture, une formalisation symbolique. »

12. Sur cette séquence onomastique, cf. Bricault (2005).

13. Cf. Parker (2017a), Smith (2016), et notre review article « Mapping ancient gods. Naming and embodiment beyond "anthropomorphism". A survey of the field in echo to the books of M.S. Smith and R. Parker », sous presse dans la Mediterranean Historical Review. 
concret ou imaginaire, d'où la divinité provient ou qu'elle habite, sur lequel elle est compétente, à moins qu'elle n’y ait manifesté sa puissance par une épiphanie. Tantôt on a recours à un toponyme ou à une qualification topographique (par ex. « de la montagne »), tantôt on utilise un substantif ou un adjectif dérivé, voire une phrase verbale ou nominale. Comment interpréter les infimes nuances qui distinguent une «Astarté de Paphos » (nom propre + toponyme) d'un « Melqart Baal de Tyr» (nom propre + titre + toponyme) ${ }^{14}$, ou un dieu Chthonios d'un dieu Katachthonios? L'espace, on le constate aisément, renvoie à une pluralité d'échelles et de réalités; il est volontiers polysémique (Polias par référence à la polis, « cité », mais aussi l'akropolis, "l'Acropole ») $)^{15}$ et charrie diverses connotations temporelles ou rituelles. Des remarques analogues peuvent être formulées au sujet d'autres catégories servant à qualifier, décrire, exalter et distinguer les dieux : les fonctions, les qualités, mais aussi les objets, les animaux, les végétaux, les personnes, ou encore les connotations physiques ou perceptives, etc., que chaque langue exprime selon son « génie » propre et dont chaque acteur ou agent social s'empare en fonction de motivations et de finalités propres.

La nouvelle terminologie que nous avons proposé d'adopter — une séquence onomastique formée de divers éléments - est plus souple et plus englobante à la fois, ce qui représente un double avantage pour l'enregistrement dans la BDD et qui neutralise, dans une certaine mesure, les différences foncières entre le grec et le sémitique. Par-delà les questions pratiques, la notion de «séquence/formule onomastique » entend souligner le fait qu'on a affaire à un véritable langage, avec sa grammaire et sa syntaxe, permettant de construire, d'énoncer, de catégoriser et de classer des entités divines relevant à la fois de l'un et du multiple, du singulier et du pluriel, au sein de réseaux sans cesse reconfigurés en fonction des contextes d'usage. L'approche mise en œuvre dans le cadre du projet MAP s'apparente en outre, à l'échelle de l'Antiquité, à une démarche de type big data, puisque la BDD a vocation à abriter toutes les séquences ou formules onomastiques attestées dans les sources retenues, ce qui représente assurément des dizaines de milliers d'attestations.

L'ensemble des enjeux que l'on vient de pointer invite à ne pas simplifier l'approche des systèmes de dénomination du divin, mais à relever le défi d'une complexité structurelle et pragmatique, que le comparatisme contribue à pénétrer. La réflexion sur les «bonnes catégories » pour l'approcher, ouverte avec la proposition explicitée ci-dessus, destinée à susciter le débat et non pas à le verrouiller,

14. Dans les textes ouest-sémitiques, on peut distinguer quatre formes d'associations entre un Divine Name (DN) et un Geographical Name (GN), à savoir : 1. «DN de GN »; 2. «DN à/dans GN »; 3. «DN qui habite/est établi (à) GN »; 4. «DN maitre(sse) de GN ». Cf. SмITH (2016), p. 71-77, avec les références antérieures.

15. Sur (Athéna) Polias, cf. Paul (2016); voir aussi Lebreton (2015) sur (Zeus) Polieus. 
constituera dès lors un fil rouge du projet MAP tout au long de son déploiement; elle représente aussi un enjeu majeur pour la validité de la nouvelle BDD.

\section{2. À la recherche des « bonnes catégories »}

En mettant en évidence la distinction entre les appellations divines permettant de s'adresser aux dieux et celles employées pour y faire référence, Robert Parker a contribué, une fois encore, à faire avancer la question ${ }^{16}$. Il est donc utile de revenir sur les différentes pragmatiques auxquelles peuvent répondre diverses formes de dénominations divines : en explorant le champ des possibles en la matière à partir de la proposition de R. Parker, il est possible de dissiper quelques confusions catégorielles.

À cet effet, un rapide détour par la terminologie grecque est nécessaire, ne serait-ce que pour rappeler qu'elle n'est que de peu de secours pour construire des catégories onomastiques solides — même dans un champ spatio-temporel restreint, voire au sein d'une seule et même œuvre. Nous ne sommes pas les premiers à affirmer que les deux grandes catégories d'épithètes divines, à savoir poétiques et cultuelles (le terme d'« épiclèses » étant réservé à ces dernières), sont étiques et non émiques, autrement dit que les mots grecs qui les recouvrent (epikèesis ou epónumia) : 1. ne sont pas spécifiques à l'onomastique divine; 2. sont interchangeables entre eux et ne correspondent à aucune des catégories susdites. On peut même ajouter qu'il n'y a pas de témoignage sûr d'une catégorie indigène d'épithète divine strictement distincte des autres éléments onomastiques, en particulier des théonymes, comme l'usage assez fluide d'onoma le laisse penser. Gageons qu'une étude systématique des termes grecs nommant les noms (divins comme non divins), tels que onoma, epikềsis, epōnumia, prosēgoria, etc., qui reste à faire, prouvera un jour ces présomptions. Quelques exemples bien choisis suffisent d'ores et déjà à en suggérer la validité ${ }^{17}$. Quand Thucydide, en l'espace de quelques lignes de son Archéologie (I,

16. Parker (2017a), p. 1-2, complétant ainsi PArker (2003). Calame, à paraître, prolonge cet axe de réflexion à partir de l'étude des hymnes de cultes : selon lui, les dénominations et qualifications utilisées pour faire référence aux dieux (notamment dans le cadre du récit) deviennent, respectivement, par l'invocation, des nominations et appellations en acte.

17. Une analyse exhaustive des quelques milliers d'occurrences de ces termes dans les sources issues de la tradition manuscrite serait fastidieuse et n'apporterait probablement guère plus que ce que tendent à indiquer quelques sondages, outre les extraits de Pausanias et Thucydide cités ici. Chez Homère, epōnumia est absent (comme dans l'ensemble de la littérature archaïque d'ailleurs) et epikelesis renvoie exclusivement à des noms alternatifs de mortels (Il. VII, 138; XVI, 177; XXII, 506) ou d'astres (Il. XVIII, 847 et XXII, $29=$ Od. V, 273), si l'on fait abstraction de l'Hymne homérique à Apollon (385-387), où c'est à l'épithète divine Telphousios que se rapporte ce terme. Si Hérodote utilise bien plus souvent epönumiè qu'epikềsis, il emploie néanmoins l'un comme l'autre de ces deux termes pour désigner aussi bien des noms de réalités physiques (I, 14; IV, 182) ou humaines (I, 94; I, 114), que des attributs onomastiques divins (II, 42; I, 19), dans tous les cas dans des contextes de référence. Par conséquent, si dans le célèbre épisode où Crésus invoque Zeus Katharsios, Epistios et Hetairèios, il est possible de détecter une nuance 
3, 2), évoque le nom de l'Hellade successivement comme un ounoma, une epiklèsis et une epōnumia ${ }^{18}$, il laisse entendre que ces trois termes sont, sinon des synonymes, du moins des équivalents interchangeables, puisqu'il les emploie indifféremment (pour éviter les répétitions?); a fortiori, il suggère (là encore, indirectement, en creux) qu'ils n'ont pas de connotation « technique » spécifique pour désigner telle ou telle catégorie de dénominations (divines ou non). À lire l'historien, on a l'impression que les attributs onomastiques peuvent être indifféremment désignés comme des noms, appellations, dénominations, vocables — tous ces noms de noms étant tenus pour équivalents (ce qui est grosso modo le cas aussi en français). Le célèbre excursus de Pausanias relatif aux attributs onomastiques de Poséidon (VII, 21, 7-9) va, à quelques six siècles d'écart et sur un sujet plus proprement " religieux ", précisément dans le même sens. Régulièrement mis à contribution dans ces discussions, il a servi d'alibi à la constitution de la catégorie d'« épiclèse » ${ }^{19}$. Pourtant, la distinction entre les différentes appellations divines que formule le Périégète ne recoupe ni celle établie entre théonymes et épithètes, ni celle qui différencie épithètes poétiques et cultuelles. L'auteur distingue en effet d'abord, au sein des onomata du dieu, ceux forgés par les poètes et les epichöria (onomata), d'usage local. Il précise enfin que - toujours au sein de ces onomata du dieu — il est des epiklèseis, des appellations, qui sont connues de tous (en l'occurrence Pelagaios, Asphalios et Hippios - cette dernière étant désignée plus loin comme un onoma), autrement dit des appellations dont la diffusion n'est cantonnée ni à des cercles savants ni à des communautés géographiquement restreintes. Par conséquent, on ne trouve pas chez Pausanias de distinction catégorielle entre théonyme et épithète (puisque onoma et epikêesis sont employés indistinctement pour désigner une même réalité),

entre kaleō et onomaz̄ō, qui dénoteraient respectivement l'invocation et l'évocation (I, 44), force est de constater que celle-ci n'est pas transposable aux substantifs correspondants. On peut en revanche synthétiser les résultats d'une enquête similaire dans les inscriptions, qui fournissent un matériel bien plus pauvre. Sur quelques dizaines d'occurrences tout au plus, les plus nombreuses se réfèrent à des sobriquets ou des cognomina de personnes; on trouve parfois des acceptions techniques, mais relevant du vocabulaire institutionnel, et non religieux (ainsi en IK.Priene 14, 1. 82, où epikêesis retranscrit probablement le latin renuntiatio, terme qui désigne la proclamation des résultats des élections). Au bout du compte, aucune ne renvoie avec certitude à quel qu'attribut onomastique divin que ce soit.

18. I, 3, 2 (à propos de l'Hellade avant la guerre de Troie) : « Ce nom (ounoma) même, me semble$\mathrm{t}$-il, ne s'employait pas encore pour la désigner dans son ensemble: avant Hellen, fils de Deucalion, il semble que cette appellation (epiklèsis) n'ait même eu aucune existence; il n'y avait que des etbnè - certains, comme l'élément pélasgique, assurant à leur propre nom (epōnumia) la diffusion la plus étendue. »

19. Ainsi (e.g.) Hornblower (2014), p. 98, à la suite de Parker (2003), p. 173, bien que ni l'un ni l'autre ne tiennent la catégorie d'épiclèse/épithète cultuelle pour absolue. 
pas plus qu'entre épithètes cultuelles et épiclèses, puisque sa catégorisation porte d'abord sur l'ampleur de la diffusion de telle ou telle appellation ${ }^{20}$.

Ceci étant dit, l'absence de terminologie technique émique n'interdit pas une pluralité effective de pragmatiques dans la façon de nommer les dieux : on peut maitriser parfaitement une langue sans avoir de connaissances théoriques sur sa grammaire; et quiconque a l'habitude de rencontrer des dénominations divines dans les sources écrites ne peut que constater que les Grecs n'employaient pas Apollon, Phoibos ou Puthios (respectivement un «théonyme », un « hétéronyme» et une " épithète cultuelle ») de façon totalement interchangeable ou aléatoire. À l'orée de notre enquête, il est sans doute trop tôt pour proposer une nouvelle typologie — si tant est que l'exercice s'avère utile, à terme. Pour l'heure, on dissipera simplement le double malentendu sur lequel repose la catégorie « épiclèse », malentendu qui réside dans les deux raisons de sa construction par les modernes à partir du terme epikelésis. Premièrement, la fréquence d'emploi du terme chez Pausanias : pour être bien réelle, elle ne correspond pas, on l'a vu, à une catégorie spécifique d'appellations, à savoir les épithètes cultuelles. Deuxièmement, le fait que ce terme dérive du verbe epikaleō et dénote ainsi l'invocation rituelle au divin ${ }^{21}$ : or, les epikèeseis que l'on trouve chez Pausanias ne font pas nécessairement référence à un contexte d'adresse. Peut-être ce terme ambigu d'épiclèse obscurcit-il plus le tableau qu'il ne l'éclaire. Quitte à allonger la terminologie, sans doute faut-il se laisser la possibilité d'identifier au cas par cas, au sein des attributs onomastiques divins, selon les contextes d'énonciation (en particulier adresse vs référence), des appellations, dénominations, épithètes cultuelles, rituelles, discursives, etc. agencées en des séquences plus ou moins longues, encapsulant telle ou telle figure divine en un ou deux mots ou, au contraire, égrenant ses attributs pour en dérouler la puissance dans toute son étendue.

\section{LES PRÉCÉDENTS : RÉPERTOIRES ET BDD}

\subsection{La nécessité d'une approche sérielle}

Le projet MAP n'a assurément pas ouvert une brèche dans un domaine jusque-là inconnu. Si l'approche systématique et comparative, ainsi que les outils que nous nous efforçons de mettre au service de l'analyse des dénominations

20. Dans une optique légèrement différente, c'est ce qu'affirmait déjà Pirenne-Delforge (2008), p. 263-264.

21. De fait, Chaniotis (1997) distingue bien deux acceptions d'epiklèsis, Anrufung d'une part et Beiname de l'autre, bien qu'il fasse découler la seconde de la première (alors que tous les Beinamen explicitement désignés comme epiklēseis par les Anciens ne sont pas nécessairement utilisés en contexte invocatoire). 
divines sont bien neufs et originaux, les questionnements qui en constituent le socle sont anciens ${ }^{22}$.

Le diagnostic formulé par le bibliste suédois Tryggve N. Mettinger résume bien l'état de l'art à la fin du $\mathrm{xx}^{\mathrm{e}}$ siècle : "When one considers the profits to be derived from a study of the divine names versus other conceivable strategies for the investigation of the Israelite understanding of God, it is quite astonishing how small a role such studies have played in the research into Old Testament theology in general and the understanding of the Israelite view of God in particular ${ }^{23}$.» Cependant, si l'on adopte un point de vue hellénocentré, le constat est moins sévère.

En effet, l'approche sérielle telle que nous la promouvons à travers la BDD MAP est déjà présente dans les listes détaillées de cognomina des dieux païens dressées au cours des $\mathrm{XVI}^{\mathrm{e}}-\mathrm{XVIII}{ }^{\mathrm{e}}$ siècles (et encore au début du $\mathrm{XIX}^{\mathrm{e}}$ ) par divers érudits, qui s'enracinent dans une conception chrétienne de l'epiklèsis, cette forme liturgique de l'invocation de Dieu/des dieux ${ }^{24}$. Au XIX ${ }^{e}$ siècle, avec l'émergence des sciences de l'Antiquité, l'orientation devient moins théologique et davantage philologique. La constitution de grands répertoires de cognomina ou d'epitheta deorum s'accompagne d'une réflexion mettant en avant la créativité littéraire (" poiétique ») des Anciens et l'ancrage "mythologique », bien plus que cultuel, des épithètes recensées. La documentation épigraphique est d'ailleurs fort peu prise en compte. Il n'empêche que le caractère systématique de ces travaux indique la voie à suivre : repérer, analyser, mettre en série ${ }^{25}$.

En 1896, avec les Götternamen d'Hermann Usener, les noms divins sont, pour la première fois, vraiment pris au sérieux ${ }^{26}$. Loin d'être de simples ornements poétiques, ou des essais infructueux pour approcher le vrai dieu, ils éclairent les modes de classification des dieux, leurs modes d'action et leur histoire. En distinguant plusieurs stades dans l'histoire des dieux (en homme de son temps) - Augenblicksgötter, Sondergötter, Personalgötter —, Usener défend une approche profondément dynamique des systèmes de dénomination, sans prétendre traiter systématiquement les données disponibles. En articulant représentation du divin et système de nomination, ainsi que pensée religieuse et action rituelle, dans une perspective généreusement comparatiste, il ouvre la voie à l'étude des noms

22. Pour un état de l'art plus détaillé qui ne concerne pas seulement la mise en série, mais les dénominations des dieux en général, voir BONNET et al. (2018), p. 570-578 et Gagné (sous presse).

23. Mettinger (1988), p. 6.

24. Ainsi par exemple le travail pionnier de Julien DE HAVReCH, De cognominibus deorum gentilium, paru en 1541. Pour un état de la question approfondi sur les recherches antérieures au XIX ${ }^{e}$ siècle, cf. GAGNÉ (sous presse).

25. Wentzel (1889). Bruchmann (1893). Preller - Robert (1894), p. 941-960 («Register der Beinamen »). Carter (1902).

26. Cf. SCHeid - Svenbro (2005). 
divins comme éléments constitutifs du langage du polythéisme. Ernst Cassirer ${ }^{27}$ développera ce volet en 1925 avec Das mythische Denken, délibérément rédigé dans les pas d'Usener en recourant, comme lui, à la philologie pour pénétrer le devenir inconscient des cultures.

À partir de la fin du XIx ${ }^{\mathrm{e}}$ et du premier tiers du Xx ${ }^{\mathrm{e}}$ siècle, l'horizon théologique de la religion grecque cède le pas à l'étude du rituel qui en vient à primer sur le mythe ${ }^{28}$, en particulier parmi les savants britanniques (la Myth and Ritual School) et français, influencés par l'école durkheimienne. Avec Louis Gernet et JeanPierre Vernant ${ }^{29}$, on abandonne définitivement l'approche essentialiste des dieux désormais appréhendés dans toute leur complexité intrinsèque, comme «système de notions », et relationnelle, au sein de la «société des dieux». Les noms et les épithètes dessinent des puissances plurielles, et non des "personnes » définies par une généalogie et des traits spécifiques leur appartenant en propre. Ils sont utilisés selon des dynamiques sociales, au sein de communautés données, Athènes fournissant à cet égard un terrain d'analyse privilégié. Les ensembles de dieux et de déesses, de rites et de mythes sont interprétés à la lumière de logiques classificatoires non pas fixes mais déterminées par les contextes d'énonciation.

Parallèlement, Milman Parry, en travaillant sur le langage de l'épopée, identifie l'épithète homérique comme la clé de voûte d'un système compositionnel complexe et plus ou moins fluide à la fois ${ }^{30}$. La mécanique combinatoire qu'il révèle, flexible et économe, avec ses nombreuses reconfigurations possibles entre tradition et création, n'est pas sans parallèle avec la sphère des performances rituelles. Le recueil des Epitheta deorum apud Homerum de James H. Dee, paru en $1994^{31}$, constitue un autre jalon intéressant pour éclairer la démarche de MAP.

Il faut cependant attendre la fin du $x^{\mathrm{e}}$ siècle et les travaux de Pierre Brulé pour que les formes d'expérimentation dans le champ des pratiques onomastiques grecques soient pleinement considérées comme un matériau à exploiter à grande échelle. Il lance à Rennes la première base de données des épiclèses grecques $(B D E G)^{32}$ et en explicite les fondements théoriques et méthodologiques dans un article séminal datant de 1998, publié dans ces colonnes ${ }^{33}$. Il dessine plus clairement encore le cahier de charges, dans l'introduction au volume collectif paru en 2005,

27. Cassirer (1925) [1973].

28. Pour l'analyse de la dialectique mythe-rite chez Cumont, pour prendre un exemple, voir Bonnet (2007).

29. Gernet - Boulanger (1932) [1970], p. 221-231. Vernant (1965) et (1974). Cf. PirenneDelForge - SCHEID (2017).

30. Parry (1928a et b) et (1971).

31. Dee (1994).

32. URL : https://epiclesesgrecques.univ-rennes1.fr/ (09/18). Cf. ci-dessous p. 281-282.

33. BRULÉ (1998). 
Nommer les Dieux. Théonymes, épithètes, épiclèses dans l'Antiquité34 — qui consacre la « triade » théonymes, épithètes, épiclèses — en affrontant la question : « Comment dire le divin? ». La notion de " paysage divin des épiclèses » lui permet de cerner les contours d'un «territoire » divin, intelligible et plus ou moins partagé, qu'il convient de concevoir comme un réseau balisé par les noms attribués aux dieux. Selon lui, une enquête à grande échelle sur ces noms en contexte s'impose, en tant que clé d'accès au langage du polythéisme, à la loi de l'offre et de la demande qui régule les relations hommes-dieux. La continuité qu'instaure le projet MAP est donc évidente et revendiquée.

Dans les mêmes années, Robert Parker cible « a central but little discussed aspect of Greek polytheism ", à savoir "The Problem of the Greek Cult Epithet » ${ }^{35}$. A-t-il raison de penser que "perhaps the extraordinary infrequency, amid all the huge literature that exists on Greek religion, of theoretical discussions of the cult epithet as a category, is the product of a suspicion that there is indeed nothing illuminating to be said except about particular examples »? Son passionnant ouvrage de 2017, Greek Gods Abroad. Names, Natures, and Transformations, apporte un démenti éclatant au soupçon formulé en 2003. Néanmoins, là où Parker a raison, c'est lorsqu'il affirme qu'il ne peut y avoir de « science » des noms divins qu'empirique, c'est-à-dire solidement adossée à l'étude des cas particuliers, tous les cas particuliers. La BDD MAP repose précisément sur cette conviction. Or, la prise en compte croissante des manières de nommer les dieux comme clé d'accès au fonctionnement du polythéisme hellénique, mais aussi d'autres systèmes religieux, y compris le « monothéisme » hébraïque, nécessite un double effort : d'une part, définir une terminologie et fixer des catégories adéquates sur le plan heuristique et herméneutique, capables notamment de rendre compte de la complexité des informations contenues dans les sources sans céder à la tentation d'une lecture chaotique et aléatoire; d'autre part, se donner les moyens d'un repérage et d'un enregistrement exhaustifs des données disponibles à ce jour ${ }^{36}$ pour assurer des analyses aussi fondées et fiables que possible, et pour ouvrir les portes à une cartographie multifactorielle du divin profondément renouvelée. Sur ce plan, le projet MAP n'est pas sans prédécesseurs. Deux étapes s'imposent à notre attention : un fichier inédit de données épigraphiques réalisé à la fin du XIX ${ }^{\mathrm{e}}$ siècle et la plus récente $B D E G$ de Rennes.

34. BeLAyche et al. (2005).

35. PARKER (2003).

36. Personne n'ignore évidemment que ces données sont le fruit de trouvailles conjoncturelles, en constant devenir, partielles par définition. Tout travail sur l'Antiquité est soumis à ces contraintes. 


\subsection{Le fichier Epitheta deorum dearumque de Friedrich Hiller von Gärtringen}

L'Akademie der Wissenschaften de Berlin conserve dans ses archives un fichier attribué à Friedrich Hiller von Gärtringen (1864-1947) et intitulé Epitheta deorum dearumque ${ }^{37}$. Dans ce répertoire, l'épigraphiste allemand a consigné l'ensemble (?) des attestations épigraphiques d'épithètes divines présentes dans le Corpus Inscriptionum Graecarum (CIG, 1828-1877), selon toute probabilité au moment où il travaillait lui-même à l'édition de plusieurs volumes des Inscriptiones Graecae $(I G)^{38}$. Le fichier a manifestement été complété par un(e) collègue, d'identité inconnue, qui a intégré les données issues du dépouillement des Inscriptiones Graecae antiquissimae praeter Atticas in Attica repertas (IGA), publiées par Hermann Roehl (1851-1923) en 1882, et des volumes 9, 10 et 11 du Supplementum Epigraphicum Graecum (SEG), parus entre 1938 et 1954. La même méthode de travail et de classement que celle établie par Hiller von Gärtringen a été suivie ${ }^{39}$.

Le fichier prend la forme d'une boite en carton d'environ $30 \times 10 \mathrm{~cm}$ contenant quelque 2132 fiches en papier fin d'environ $10 \times 3 \mathrm{~cm}$. Chacune de ces fiches répertorie une séquence onomastique divine attestée épigraphiquement. Sont à chaque fois consignés, au recto, le texte de l'attestation et la référence bibliographique de l'inscription ainsi que, au verso, d'éventuelles précisions sur l'identification de la divinité concernée ou sur la lecture de l'inscription. La refonte du CIG dans des corpora épigraphiques plus récents rend évidemment un tel outil obsolète. Son intérêt historiographique n'en est pas moins grand : par son mode d'organisation, ce fichier rend compte de l'état de la réflexion de Hiller von Gärtringen dans le traitement des épithètes divines et, on le verra, des séquences onomastiques. C'est donc sur les choix adoptés par l'épigraphiste allemand pour relever ce défi qu’il est utile de se pencher ici.

37. Ce fichier nous a été prêté par Klaus Hallof, directeur des Inscriptiones Graecae de l'Académie de Berlin, ce dont nous lui sommes extrêmement reconnaissants. Il sera restitué aux collègues avec le répertoire Excel des fiches, ainsi qu'un fichier de numérisation de celles-ci afin d'en assurer la préservation numérique. Pour un état des lieux des archives des $I G$, cf. Summa (2012).

38. Les lignes qui suivent doivent beaucoup à l'admirable travail de numérisation (sous forme de tableur Excel) et d'étude du fichier effectué par Marta Tacchetto, étudiante en Master de l'université de Padoue, en mobilité Erasmus à l'université Toulouse - Jean Jaurès, dans le cadre d'un stage de Master auprès du projet MAP. Nous lui sommes très reconnaissants d'avoir accepté de mettre à notre disposition, pour cet article, son rapport de stage.

39. On peut supposer que Hiller von Gärtringen a constitué le fichier au plus tard au moment où il s'est attelé à l'édition des volumes des IG XII durant les années 1890, puisqu'il ne fait référence qu'au CIG. Étant décédé en 1947, il n'a pu dépouiller les volumes 10 et 11 du SEG (parus en 1949 et 1950-1954) : les données issues de ceux-ci, de même que du volume 9 (paru en 19381944) et des $I G A$, ont donc été intégrées par un successeur (ce que confirme la différence de graphie) après la mort de Hiller von Gärtringen, sans doute à partir de 1954, si l'on considère que cette seconde phase a été continue. 
Les fiches sont soumises à un double classement alphabétique. Le premier porte sur les théonymes des divinités répertoriées, qui sont ainsi réparties en 60 rubriques séparées par des intercalaires. Au sein de chacune de ces rubriques, les fiches sont ensuite rangées suivant l'ordre alphabétique de chaque épithète. De ce point de vue, la logique est analogue à celle qui préside à l'établissement de la section Nomina sacra de l'index d'un corpus épigraphique. Au-delà de sa fonction première - permettre à l'utilisateur de retrouver aisément les occurrences de tel item indexé _- cet outil donne un aperçu global de la répartition quantitative des épithètes entre les différentes divinités. Sur ce plan, le fichier Hiller von Gärtringen est particulièrement parlant, compte tenu du nombre d'occurrences qu'il consigne, mais aussi de sa matérialité ${ }^{40}$ : une simple vue en contre-

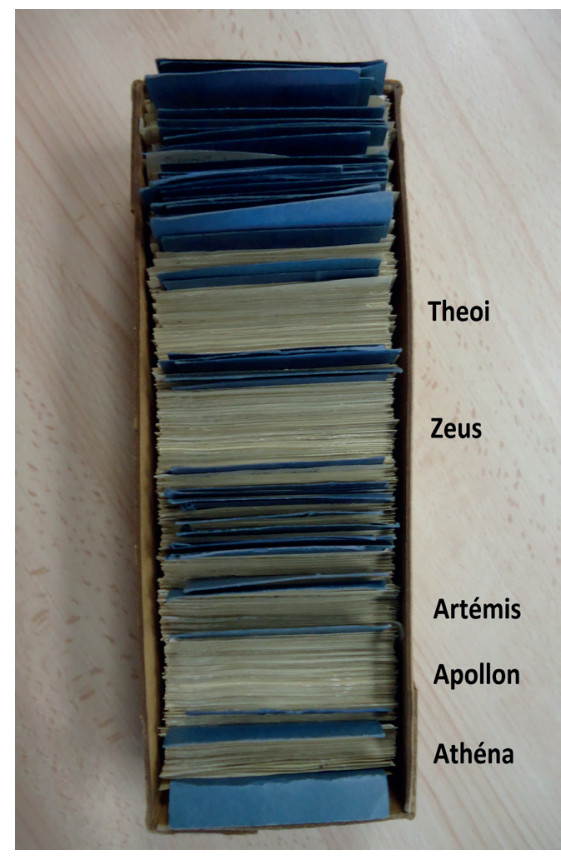

FIG. 1. Le fichier Hiller von Gärtringen : aperçu de la répartition des fiches. plongée du fichier offre une vue synoptique sur la richesse respective des différentes puissances divines en épithètes. On constate ainsi rapidement que, avec les dieux désignés de façon générique (les Theoı) ${ }^{41}$, Zeus est le plus abondamment doté en la matière, suivi d'Apollon, Artémis, Athéna, etc. En dépit de (ou peut-être grâce à) son caractère tout à fait empirique, et compte tenu de la masse critique des données prises en compte, ce répertoire instantané n'en a pas moins une valeur démonstrative certaine (Fig. 1). Gageons que le tableau impressionniste qui s'en dégage sera confirmé et raffiné, avec plus de précision et de nuances, par l'utilisation d'outils d'analyse quantitative des données dignes de ce nom, comme le projet MAP se propose de le faire ${ }^{42}$.

L'unité d'enregistrement adoptée par Hiller von Gärtringen (la fiche) ne se prête du reste qu'à un traitement quantitatif très élémentaire. En effet, à chaque fiche correspond une occurrence documentaire attestant de la combinaison d'un théonyme donné avec une épithète donnée. Ainsi par exemple, au sein de la rubrique consacrée à Zeus, 40 fiches concernent l'épithète Olumpios. Toutefois, lorsqu'une séquence onomastique complexe combine plusieurs épithètes, une

40. Sur l'histoire de la fiche érudite, voir BERT (2017).

41. De fait, les dédicaces adressées aux Theoi Katachthonioi (autrement dit les Mânes), qui figurent en tête des épitaphes, forment l'écrasante majorité des occurrences réunies dans cette rubrique.

42. Cf. infra, p. 282-293. 
fiche est constituée pour chacune d'entre elles. Par conséquent, plusieurs fiches peuvent renvoyer à une même attestation épigraphique : ainsi par exemple, une dédicace à Apollon Prostaterios Apotropaios Aguieus ${ }^{43}$ fait l'objet de trois entrées, une pour chaque épithète (Fig. 2). Cette méthode d'indexation empêche tout véritable traitement quantitatif des données, pour lequel il aurait fallu qu'à une occurrence documentaire corresponde une seule et même fiche. La finalité envisagée par Hiller von Gärtringen était plutôt, tout simplement, de faciliter l'accès à chacune des épithètes divines.

Au-delà de cette question quantitative, un certain pragmatisme dans l'enregistrement des données est à

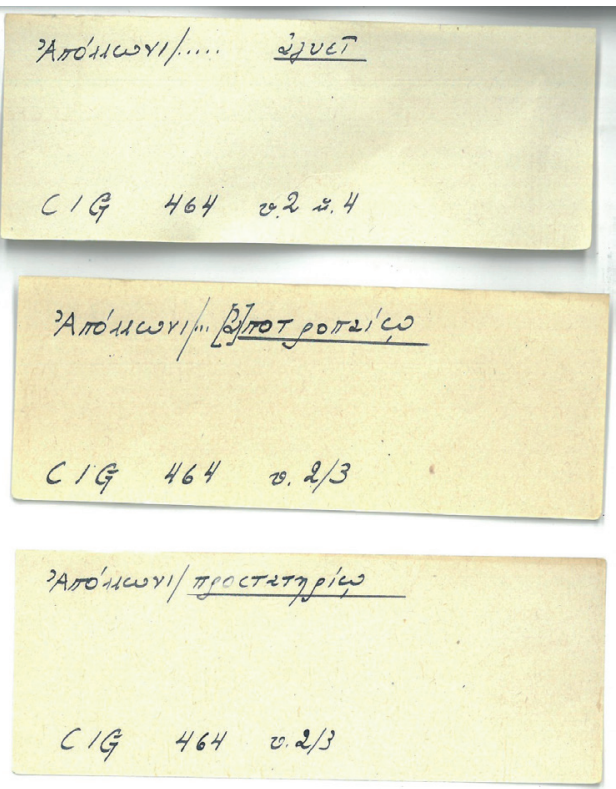

FIG. 2. Les trois fiches retranscrivant la dédicace $C I G 464\left(=I G I^{3} 4,953\right)$. l'œuvre: on trouve en effet répertoriés dans le fichier toute sorte d'attributs onomastiques - des hétéronymes et des épithètes "poétiques » tout autant que des épithètes cultuelles — au sein de séquences onomastiques de taille et de composition variées. Il n'en reste pas moins que la partition théonyme/épithète détermine l'architecture du fichier, ce qui a poussé son auteur à faire des choix. Dès lors, si les différentes épithètes d'une séquence onomastique donnée se voient chacune consacrer une fiche, il n'en va pas de même des différents théonymes qui peuvent parfois se trouver combinés. Ainsi, par exemple, Zeus Asklapios (CIG 1198) et Zeus Sarapis (CIG 7042 e.g.) sont-ils uniquement enregistrés dans les rubriques consacrées, respectivement, à Asclépios et Sarapis, mais non pas à Zeus — l'élément Zeus étant, dans ces cas, considéré (peut-être à juste titre) comme une sorte de titre ${ }^{44}$. On note cependant que cette logique est à géométrie variable, puisque le syntagme Zeus Malbachos (CIG 4450 e.g.) figure à la fois dans les rubriques «Zeus » et «Malbachos». Ces incohérences dans la démarche semblent révélatrices de la difficulté à appréhender l'ensemble du champ des possibles en matière d'onomastique divine et de maintenir un équilibre entre efficacité de l'indexation et fidélité de la retranscription des séquences onomastiques divines dans tout leur polymorphisme. Ces difficultés ont

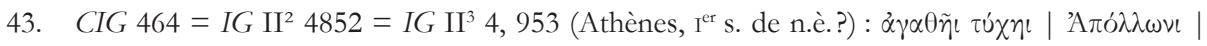

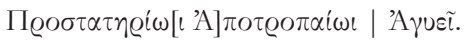

44. Sur cette question, cf. PARKer (2017b). 
aussi été abordées à Rennes un siècle environ après le fichier pionnier dû à Hiller von Gätringen.

\subsection{Des nouvelles de la BDEG}

Si la Banque de Données des Épiclèses Grecques (BDEG) initiée par Pierre Brulé au début des années 2000, et mise en ligne il y a plus de dix ans déjà, a constitué une indéniable avancée dans le traitement de ce matériau foisonnant que constituent les épithètes divines du polythéisme hellénique, elle n’a pas davantage permis de trouver cet équilibre. Il n'est pas utile d'exposer à nouveau les principes directeurs de cet outil, déjà présentés entre ces pages ${ }^{45}$. Rappelons simplement que la $B D E G$ visait à enregistrer toutes les épithètes cultuelles attestées dans l'ensemble des sources écrites grecques (de fait, l'effort a essentiellement porté sur les sources littéraires et épigraphiques). Sa structure est la suivante : une fiche correspond à une combinaison théonyme + épithète dans un lieu donné (l'échelle de référence envisagée étant la cité). Outre la simplicité dans les métadonnées qu'elle permettait de consigner (toutes les informations relatives à la source, en dehors de sa datation, sa référence bibliographique et son type - épigraphique, littéraire, numismatique ou papyrologique — - mais aussi au contexte ou aux agents, étaient consignées dans un même champ de commentaire), on prend aujourd'hui la mesure de ses limites conceptuelles et techniques. Les deux principales résident dans la structure même de la fiche d'enregistrement. En premier lieu, le choix de prendre pour unité de base le lieu dans lequel une combinaison théonyme + épithète est attestée empêche tout traitement quantitatif des données digne de ce nom, puisqu'à une même fiche peuvent correspondre une ou plusieurs occurrences documentaires $^{46}$. Deuxièmement, la présence de champs strictement dévolus aux théonymes («Divinité ») et aux épiclèses (« Épiclèse 1 » et « Épiclèse 2 » si besoin) rendait difficile la saisie de séquences onomastiques divines sortant du système de dénomination nom-épithète, telles que Zeus Hêlios Megas Sarapis évoquée plus haut ${ }^{47}$. Ces limites (évidemment plus faciles à identifier a posterion) auraient

45. Brulé - Lebreton (2007).

46. De fait, la fiche était conçue pour n'enregistrer, au plus, que deux attestations documentaires («Source 1 » et «Source 2 », en principe la plus ancienne et la plus tardive) d'une même combinaison théonyme + épithète dans un lieu donné. Outre le fait que l'entité «lieu » peut être envisagée avec un degré de précision variable (le plus souvent une cité, mais aussi un dème, un site, un sanctuaire donné), l'abondance et surtout la variété des attestations ont pu pousser certains rédacteurs de fiches à enregistrer plus de deux sources pour une même combinaison théonyme + épithète en un même lieu. La BDEG y a gagné en richesse documentaire ce qu'elle a perdu en possibilité de traitement quantitatif des données.

47. Là encore, les rédacteurs ont dû bricoler pour enregistrer de telles séquences, en désignant un des différents attributs onomastiques comme théonyme (alors saisi dans le champ «Divinité ») et/ou en établissant plusieurs fiches à partir d'une même attestation, comme pouvait le faire Hiller von Gärtringen. 
nécessité une refonte trop importante pour que cet outil dépasse ce qu'il était, à savoir — ni plus, ni moins - un répertoire dynamique permettant de trier et de croiser les données, d'opérer des requêtes selon des critères variés (et notamment géographiques), possibilités que n'offraient pas le fichier Hiller von Gärtringen (et encore moins les répertoires et index papier). En tant que tel, il peut toujours rendre d'importants services à la communauté scientifique, d'autant plus qu'il a continué à être nourri ${ }^{48}$. Mais il a vocation à être remplacé par — à défaut d'avoir pu être refondu dans ${ }^{49}$ — la BDD MAP, dont l'élaboration a précisément tenu compte - dans l'objectif de les dépasser — des limites de la BDEG.

\section{LA BDD MAP : QUELLES PLUS-VALUES?}

\subsection{Le modèle conceptuel de données}

Le projet MAP marche sur deux jambes. D'une part, il envisage, dans les séquences onomastiques, les éléments partagés ou non comme traces de la structuration des ensembles de dieux attestés dans des espaces-temps donnés (des "panthéons », pourrait-on dire); d'autre part, il se focalise sur l'agentivité humaine dans la composition et l'usage des séquences en relation avec divers paramètres contextuels. La BDD a donc vocation à enregistrer les informations susceptibles de permettre des requêtes/enquêtes sur ces deux voies privilégiées, mais non pas exclusives. La conception de la BDD a dès lors aussi consisté à trouver un équilibre entre deux pôles; cibler et servir les objectifs précis et ambitieux du projet MAP, d'une part, éviter de surcharger la BDD d'informations certes intéressantes, mais secondaires par rapport à la focale de notre recherche, d'autre part. Pour prendre un exemple, les données prosopographiques ${ }^{50}$, comme la généalogie d'un dédicant, n'ont pas leur place dans la BDD MAP. La lourdeur d'un répertoire tentaculaire et labyrinthique rendrait sa consultation lente et malaisée; il perdrait en efficacité et lisibilité. Pour s'en prémunir, l'essentiel est de tenter de prévoir les requêtes que les futurs usagers de la BDD MAP pourraient exprimer, autrement dit concevoir un outil apte à prendre en compte un ensemble de questionnements à la fois large et pertinent. Au terme de longs mois de réflexion et de tests, nous

48. Notamment tant que la BDD MAP ne sera pas complète. On peut toujours la consulter à l'adresse suivante : https://epiclesesgrecques.univ-rennes1.fr/accueil.php.

49. Une convention a été signée en 2017 entre le LAHM (ex-Crescam) et l'université Toulouse Jean Jaurès pour permettre le reversement de la BDEG dans la BDD MAP, mais les structures des deux outils et le format des données sont à ce point différents qu'il est plus simple de réenregistrer les données que de concevoir un programme pour transférer de l'une à l'autre les informations, les reformater, les contrôler, les corriger et les compléter.

50. Nous sommes cependant en contact étroit avec l'équipe constituée autour de Karine KarilaCohen (université Rennes 2), qui travaille sur la prosopographie athénienne, pour tenter de mutualiser certaines données. 
espérons être arrivés à concevoir et produire un outil original et performant ${ }^{51}$, capable de traiter la masse considérable de données à enregistrer et de répondre à une panoplie de questionnements simples mais essentiels, en croisant les champs soumis à requête : qui s'adresse au(x) dieu(x) en mobilisant tel ou tel attribut onomastique (les agents)? quand (datation; occasion)? où (localisation)? dans quel contexte matériel et rituel? pour quelles finalités? quels dieux pour quels éléments (et vice versa)? quels éléments dans quelle séquence?

Ces interrogations, et bien d'autres, peuvent engager des enjeux « macroscopiques » que l'on peut regrouper en deux ensembles. Sur le versant « théologique », les données mises à disposition dans la BDD MAP permettront d'aborder la question lancinante et pourtant si centrale : qu'est-ce qu'un dieu? Il n'est assurément pas la somme des noms qu'on lui attribue, mais ceux-ci esquissent un micro-réseau à l'intérieur du macro-réseau que serait tel ou tel " panthéon », conçu comme un ensemble conjoncturel de puissances divines. "Qu'est-ce qu'un panthéon? » est une autre question qui s'impose, tout comme : en quoi et comment les données prises dans leur ensemble font-elles système? Les notions de poly- et de monothéisme gardent-elles leur pertinence à la lumière des pratiques onomastiques? Comment l'espace et le temps influencent-ils les représentations onomastiques du divin? Observe-t-on des transformations qui conduiraient vers des entités du type big gods? Sur le versant pragmatique, par ailleurs, en quoi et comment les manières de nommer les dieux participent-elles d'une interaction à visée « utilitaire »? Quelle péréquation observe-t-on entre les noms et les bienfaits attendus ou obtenus? Quelle gestion empirique de l'énorme réservoir d'attributs onomastiques? Selon quelles logiques les acteurs posent-ils des choix, entre tradition et créativité?

Dans l'optique d'élaborer l'outil le mieux adapté à soutenir de tels questionnements, nous avons décidé de construire une base de données relationnelle en langage PostgreSQL, bilingue français/anglais. Le recours au langage XML (notamment Epidoc) et au web sémantique, un temps envisagé, n'a pas été retenu : en effet, il ne s'agit pas de proposer une édition numérique de sources écrites, mais un outil de référencement d'un matériau de nature variée permettant son traitement statistique et cartographique au moyen d'outils de webmapping et d'analyse de réseaux. Conformément aux engagements de l'ERC, nous avons opté pour un logiciel open source et la BDD sera entièrement en open access ${ }^{52}$. Une interface

51. Nous tenons à souligner ici le travail considérable (et la patience!) de notre ingénieur de recherche, Antoine Laurent, de nos stagiaires informaticiens, Maxime Savaignac (IUT Toulouse) et Stanislas Köhler (université Toulouse - Jean Jaurès), ainsi que l'expertise de Romain Gonçalves, de la société 3RGOTECH. Un grand merci aussi à Guillaume Cabanac, informaticien de l'université Toulouse - Paul Sabatier, laboratoire IRIT, qui nous a accompagnés de ses précieux conseils.

52. On y accèdera — à partir de fin 2020 selon nos prévisions — par le biais de notre site web, moyennant une inscription (libre) comme usager. L'équipe du projet MAP garde en revanche le contrôle du back office. 
de webmapping en PostGIS est en cours de test, tandis que l'interface de statistique et d'analyse de réseaux est en cours d'élaboration (avec Gephi ou UCINet) ${ }^{53}$. L'hébergement de cet ensemble par la TGIR ("Très Grande Infrastructure de Recherche", CNRS) Huma-Num garantit une maintenance d'une durée de trente ans.

La structure de la BDD repose sur trois niveaux d'enregistrement des données : une «source » (niveau 1) contient une ou plusieurs «attestation(s) ", c'està-dire une ou plusieurs chaînes onomastiques (niveau 2), chacune étant composée

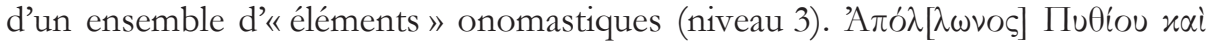

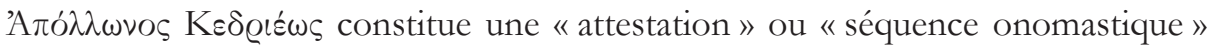
grecque, tout comme lrbt ltnt pn bll w l'dn lb'l hmn est une "attestation» ou «séquence onomastique » punique. La première comprend 4 éléments, la seconde en comporte 7 (les conjonctions de coordination n'étant pas prises en compte comme éléments). Chacune provient d'une «source » qui pourrait éventuellement contenir d'autres «attestations » ou « séquences », que l'on enregistrerait séparément, tout en les rattachant à la même fiche « source».

À ces trois niveaux d'enregistrement correspondent les trois tables principales du modèle conceptuel de données, répondant à une progression logique (1 contient 2 qui contient 3) et elles-mêmes reliées à d'autres tables secondaires propres ou partagées. Ainsi la table «bibliographie » contient-elle des références bibliographiques (enregistrées selon des normes prédéfinies) qui concernent la source dans son ensemble ${ }^{54}$ ou un élément onomastique en particulier (par exemple l'adjectif Putbios). À l'inverse, la table "pratique associée » (par exemple « libation») se rattache exclusivement à une «attestation » donnée; elle ne concerne pas les niveaux «source» ou «élément». Chacune des tables comporte un certain nombre de champs, soit en saisie libre (comme les champs de commentaire), soit, comme c'est le cas pour la plupart d'entre eux, associés à un menu déroulant contenant une liste de valeurs dans laquelle choisir afin d'assurer un enregistrement homogène des données. Pour certains champs, le choix est limité à une seule valeur; pour d'autres, on peut en combiner plusieurs. Seul un nombre limité de champs est obligatoire pour valider la saisie, le principe étant de ne pas alourdir le traitement de documents simples (ou pauvres). L'ensemble des valeurs proposées pour les différents champs forme un «dictionnaire des données » conçu en français et en anglais ${ }^{55}$. Un double Guide pour l'usager, en vue de l'enregistrement des données et de leur consultation, explicite les règles suivies ou à suivre; elles apparaittront sous la forme de fenêtres s'ouvrant au niveau de chaque champ.

53. Sur ces outils et leurs finalités, cf. infra, p. 293-294.

54. Les références aux principaux corpus épigraphiques grecs et sémitiques y sont préenregistrées.

55. La traduction des listes de valeurs est automatisée, de sorte que si l'on saisit «marbre » dans une fiche francophone, « marble » sera automatiquement enregistré dans la fiche anglophone, et vice-versa. 
L'analyse d'un document simple mais représentatif donnera sans doute un aperçu clair de la structure de l'outil et des champs qui le composent. Prenons la dédicace athénienne $I G \mathrm{II}^{3}$ 4, 1066 :

Dédicace à Artémis Dictynna. Athènes, début du $\mathrm{II}^{\mathrm{e}}$ siècle.

Base de marbre découverte «à Limnai », près du théâtre de Dionysos.

Ed. $I G I^{2}$ 4688. $I G I^{3}$ 4, 1066.

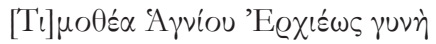

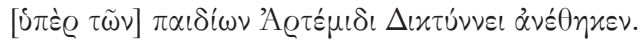

Timothéa, épouse d'Hagnias d'Erchia, a dédié (ceci) à Artémis Diktunna, pour ses enfants.

Cette inscription fera l'objet d'un enregistrement en trois temps : seront saisies les données concernant respectivement la source $\left(I G \mathrm{II}^{3} 4\right.$, 1066), l'attestation (la dédicace à Artémis par Timothéa) et la séquence onomastique divine composée de

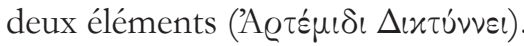

\subsubsection{La source}

Au niveau 1, dans le formulaire portant sur la source, on enregistre en particulier des informations relatives à la catégorie du texte (épigraphie, glyptique, numismatique, papyrologie, source issue de la tradition manuscrite), à son type (décret, hymne, lettre, etc.), à sa ou ses langue(s). Le matériau et le support sont indiqués avec un double niveau de précision, au moyen de listes emboîtées, ici respectivement " pierre > marbre » et « architecture > base ».

Un lien URL est donné vers une édition numérique du texte, quand elle existe (la situation dans le domaine grec étant bien plus favorable que pour le sémitique); la présence d'une iconographie est signalée et une bibliographie est fournie, en précisant l'édition de référence utilisée pour l'établissement du texte (ici $I G$ $\mathrm{II}^{3}$ 4, 1066; IG $\mathrm{II}^{2} 4688$ n'est mentionnée qu'à titre indicatif). La datation de la source est retranscrite en données chiffrées au moyen d'un post quem et d'un ante quem (ici -200 et -175$)^{56}$. Pour la localisation de la source - en deux temps si besoin : lieu de découverte, lieu d'origine — nous avons opté pour un enregistrement à cinq échelles maximum : grande région (Grèce continentale), sous-région (Attique), entité politique (Athènes), ville (Athènes), site (Théâtre de Dionysos), seule la première étant obligatoire ${ }^{57}$. On a intégré la géolocalisation à l'échelle de la ville, en ayant recours au site Pleiades (https://pleiades.stoa.org/) auquel on peut

56. Et ce, quel que soit son format: les datations « $\mathrm{VI}^{\mathrm{e}}-\mathrm{V}^{\mathrm{e}} \mathrm{s}$. », « début du $\mathrm{II}^{\mathrm{e}} \mathrm{s}$. », «429/8» ou « règne d'Hadrien» (e.g.) seront enregistrées respectivement «-600/-400», «-200/-175», «-429/-428» et «117/138». L'unité retenue pour retranscrire les «début», « milieu» ou « fin» est le quart de siècle.

57. Une valeur « Inconnue » a été intégrée à la liste des grandes régions, afin de pouvoir enregistrer les sources dont la localisation ne peut être déterminée, même à cette échelle. 
se connecter directement depuis le formulaire d'enregistrement de la source. Les champs de commentaires permettent d'apporter les précisions jugées nécessaires par le rédacteur concernant la datation, la localisation, le support, etc., notamment pour rendre compte de débats lorsque ces données sont discutées ou problématiques.

\subsubsection{L'attestation : éléments de contexte}

La deuxième table, centrée sur les «attestations», fournit une série d'informations sur la ou les séquences onomastiques (en poésie ou en prose) contenues dans la source, renseignées avec ou sans restitution, en translittération et traduction (française et anglaise). À ce niveau, l'accent est mis sur deux ensembles de données : celles qui touchent à la contextualisation de l'attestation (pas de la source dans sa totalité) et aux agents concernés. D'abord, les pratiques associées à la mention de cette séquence onomastique (ici l'offrande, explicitée par $\left.\alpha \dot{\alpha} \varepsilon \theta^{\prime} \eta \chi \varepsilon v\right)$ ), ainsi que - ces données étant absentes dans l'exemple choisi - l'occasion (mobilité, par exemple), l'éventuel matériel associé (un animal, par exemple), les éléments de localisation ou de datation (par exemple, dans un calendrier sacrificiel, la date d'accomplissement d'un sacrifice à une divinité donnée), si ces éléments sont explicités dans l'attestation. Ensuite, on renseigne le ou les agent(s), dont on retranscrit la désignation (sans prendre en compte les anthroponymes et la filiation) et que l'on distingue en fonction de leur agentivité, genre, statut, activité, localisation... Seront donc enregistrés dans le cas présent deux agents :

\begin{tabular}{|l|l|l|}
\cline { 2 - 3 } \multicolumn{1}{c|}{} & \multicolumn{1}{c|}{ Agent 1 (Timothéa) } & Agent 2 (les enfants de Timothéa) \\
\hline Désignation & Femme/épouse (gunè) & Enfants (paidiōn) \\
\hline Agentivité & Destinateur & Bénéficiaire \\
\hline Nature & Humain & Humain \\
\hline Genre & Féminin & Indéterminé \\
\hline Statut affiché & Citoyen; Familial & Citoyen; Familial \\
\hline Activité & $/$ & $/$ \\
\hline Localisation & Grèce continentale $>$ Attique $>$ Athènes $(L A C P$ 361) $>$ Erchia \\
\hline
\end{tabular}

Pour l'ensemble des champs du niveau "attestation ", on dispose de listes de valeurs, avec choix obligatoire ou non, unique ou multiple.

\subsubsection{La séquence onomastique divine et ses éléments}

Le cœur du réacteur, si l'on peut dire, c'est l'analyse des «attestations » ou « séquences onomastiques », qui constitue le troisième étage de la «fusée » MAP. 
Chaque élément constitutif d'une attestation sera enregistré une fois pour toutes dans un formulaire en tant que « lemme » ou élément invariant, accompagné d'une ou de plusieurs traductions, ainsi que de «catégories » (trois maximum) sémantiquement évoquées par l'élément indépendamment de ses contextes d'usage. L'établissement de la liste de ces catégories sémantiques a été long et difficile, soumis à la logique de l'accordéon, avant de se stabiliser autour de quarante macro-catégories comme « justice », « santé », « végétal », « social », « mobilité »... Il est très probable que cette liste ne satisfasse personne, pas même nous, tant il est impossible d'exprimer la complexité sémantique et la polysémie véhiculée par les éléments à travers quatre petites dizaines de catégories. Toutes les critiques et propositions seront dès lors les bienvenues, mais l'exercice est destiné à rester imparfait et frustrant. Il répond pourtant à une nécessité : pouvoir regrouper les éléments ou les interroger au départ de catégories qui sont autant d'entrées thématiques dans les divers axes des recherches sur l'Antiquité (par exemple, la place des pratiques sexuelles dans les cultes, l'implication des dieux dans les activités sportives, les diverses formes de mobilité ou l'exercice de la justice, etc.). Les recouvrements entre les catégories retenues sont inévitables; ils reflètent le réseau de significations multiples qui servent à parler des dieux, qu'il serait vain et contre-productif de vouloir rigoureusement compartimenter. Tout dieu couvre plusieurs sphères d'activité et toute sphère est prise en charge par une multiplicité de dieux.

Dans la table « élément », on peut rattacher un élément à un autre, par exemple dans le cas d'un hétéronyme (Pallas rattaché à Athéna), d'une variante dialectale (Pasios à Ktēsios) ou d'un élément construit sur un autre (Hēraios à Héra); de la sorte, dans les analyses statistiques ou de réseau, on ne perdra pas cette information : par exemple les formules contenant Pallas pourront être (ou non, selon les souhaits de l'usager) englobées dans le dossier " Athéna ». On renseigne aussi une éventuelle localisation contenue dans un élément (Ephesia renvoyant à Éphèse). Un champ de bibliographie et de commentaires sont disponibles.

Chaque élément dispose ainsi de sa fiche qui peut être «appelée » lorsqu'on analyse une séquence donnée, en contexte. Dans cette fiche, on précise, pour chaque élément, sa position dans la séquence, son état morphologique, son genre, son nombre, la présence d'un suffixe (pour le sémitique). Si c'est pertinent, on peut sélectionner, pour caractériser sa portée sémantique dans ce contexte précis, une des trois catégories indiquées dans le lemme (catégories hors contexte) ou bien en choisir une quatrième propre à cette attestation.

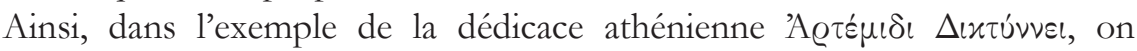
décomposera la séquence onomastique divine en deux éléments, deux substantifs

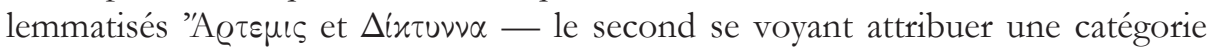
sémantique invariante, en l'occurrence pêche/chasse, en vertu de son étymologie, du reste incertaine, l'associant au filet (diktuon). Artémis n'est rattachée à aucun champ sémantique hors contexte; en revanche, dans l'analyse en contexte, pour les 
deux éléments, tant Artémis que Diktunna, la catégorie sémantique «Génération/ Croissance » sera proposée à la lumière de l'identité de la dédicante et (surtout) des bénéficiaires.

Enfin, pour tenter de comprendre les logiques qui président à la construction des puissances divines par l'acte de nomination, il ne suffit pas de photographier une attestation et chacun de ses éléments; il est indispensable d'aller plus loin en tentant de saisir les processus de dénomination et de formaliser la structure interne des attestations ou séquences onomastiques, ce qui revient à scruter la complexité inhérente à la pragmatique des noms divins. En nous inspirant de la formalisation mathématique, nous avons mis au point une méthode nouvelle pour traduire chaque séquence onomastique en une « formule » qui reflète son architecture, donc sa formation. La formule rend ensuite possible le traitement informatique, en série, des séquences onomastiques divines, donc des combinatoires dans leur ensemble (statistiques, réseaux, visualisation dynamique, etc.), ce qui serait objectivement impossible à l'échelle d'un travail individuel et manuel. C'est là qu'intervient la dimension big data du projet MAP et l'espoir de produire des résultats neufs et significatifs dont toute la communauté scientifique pourra s'emparer pour développer, au départ du traitement sériel, des analyses dûment fondées, alliant dimension structurelle et historique. Si on prend de la hauteur, on pourra mettre en valeur la double orientation des pratiques onomastiques : d'une part, la fidélité à la tradition, aux modèles, aux usages, avec certaines formules qui se répètent à l'envi des centaines, voire des milliers de fois; d'autre part, la marge de manœuvre dont dispose chaque acteur pour introduire des variantes, nuances ou nouveautés qui font sens dans un contexte donné ${ }^{58}$. Entre rigidité et fluidité, les formules onomastiques apparaitront comme le reflet de pratiques sociales multifactorielles, largement imprévisibles, et pourtant en partie normées.

\subsubsection{Les points forts de la BDD $M A P$}

L'attention portée à la spatialisation des données est un premier atout de la BDD; pour un projet intitulé Mapping Ancient Polytheisms, cela s'impose. On peut donc enregistrer des informations spatiales à quatre niveaux : à la source, à l'attestation, à l'agent et à l'élément, avec chaque fois cinq niveaux de précision et une géolocalisation. Pour la source, on distingue le lieu de découverte et d'origine; la qualification des lieux contenus dans les éléments différencie ce qui relève de la toponymie et de la topographie, du réel ou du fictif, et précise en outre la fonction des lieux désignés (politique, économique, loisir, échanges...). La richesse et la précision des informations permettront en amont une cartographie dynamique, ou webmapping, consistant en des outils de visualisation perfectionnés, qui seront

58. Sur ce plan, des recoupements sont évidents avec le projet ERC Lived Ancient Religion de Jörg Rüpke, à Erfurt (terminé en 2017), qui a souligné la part de l'individu, les processus d'appropriation et d'expérience religieuse. 
autant d'avantages pour analyser qualitativement, par le biais d'une démarche proprement historique, les configurations ou réseaux émergeant des données.

Par ailleurs, nous souhaitons fournir aux usagers de la BDD un outil apte à évaluer la qualité des données fournies. Cet outil s'applique à trois niveaux : la précision de la datation et de la localisation de la source, ainsi que la fiabilité de la lecture de l'attestation. Pour la datation de la source, l'évaluation prend la forme d'un coefficient, allant du plus précis (1, entre un et cinq ans) au moins précis (5, plus de deux siècles). Le calcul se fait automatiquement, en fonction de la date saisie par le rédacteur, c'est-à-dire de l'intervalle entre post quem et ante quem. Pour la localisation de la source, le coefficient, également automatique, va du plus précis (1, au site) au moins précis (4, à la grande région), en fonction des échelles de précision renseignées par le rédacteur ${ }^{59}$. Pour la fiabilité de la lecture de l'attestation dans sa totalité ${ }^{60}$, c'est le rédacteur de la fiche qui renseigne un coefficient, de 1 à 3 : $1=$ Lecture assurée $; 2=$ Lecture probable $; 3=$ Lecture incertaine.

Cependant, la fonctionnalité la plus originale de la BDD MAP est, du moins nous l'espérons, la «formule » que le rédacteur peut proposer à la fin de la table « attestation » pour retranscrire la composition de la séquence onomastique divine.

\subsection{Le traitement des séquences onomastiques divines}

Par sa structure même, en raison de la richesse des données relatives aux attestations des attributs onomastiques divins qu'elle permet de consigner et qui faisaient défaut à la BDEG (agents, contexte...), la BDD MAP constitue un outil d'analyse des noms des dieux et de leurs usages significativement plus puissant que ses prédécesseurs. Mais son apport le plus significatif, le plus novateur, réside dans le champ «formule » qui clôt la table «attestation » de la BDD. En effet, chaque séquence onomastique donnée sera analysée au moyen d'une formule permettant d'en retranscrire l'agencement, c'est-à-dire la façon dont les éléments qui la composent sont combinés. Contrairement au fichier de Hiller von Gätringen ou à la $B D E G$, la position des éléments et les liens qui permettent leur articulation pourront être pris en compte grâce à cette formule. Ce faisant, ils seront notamment exploitables par les outils d'analyse des réseaux qui complèteront la BDD MAP.

La formalisation des séquences permet d'encoder trois catégories d'informations relatives à leur composition :

- la position des éléments au sein de la séquence : celle-ci sera indiquée automatiquement par un chiffre $(1,2,3 \ldots)$ au fur et à mesure que les éléments sont enregistrés dans la table attestation de la BDD;

59. L'entité politique n'est pas prise en compte parce qu'elle n'est pertinente que pour le monde grec; de fait, la liste des valeurs de ce champ est tirée de l'inventaire des poleis établi par HANSEN - Nielsen (2004).

60. On notera qu'il ne s'agit pas de son état de conservation; un fragment peut présenter une lecture assurée alors qu'une attestation complète peut être de lecture incertaine. 
- les liens entre les éléments : on en distinguera quatre, indiqués chacun par un symbole différent :

\begin{tabular}{|l|l|c|}
\hline Désignation & \multicolumn{1}{|c|}{ Description } & Symbole \\
\hline Coordination & $\begin{array}{l}\text { Lorsque deux éléments sont coordonnés, typiquement } \\
\text { par la conjonction kai (en grec) ou } w \text { (dans les langues } \\
\text { sémitiques) : « et » }\end{array}$ & + \\
\hline Juxtaposition & $\begin{array}{l}\text { Lorsque deux éléments sont juxtaposés sans que l'on } \\
\text { puisse déterminer, a priori, si l'un est qualifié par l'autre : } \\
\text { c'est notamment le cas lorsque se suivent deux éléments de } \\
\text { même nature grammaticale }\end{array}$ & / \\
\hline Qualification & $\begin{array}{l}\text { Lorsqu'un élément est qualifié par un autre, typiquement } \\
\text { (mais pas seulement), un substantif par un adjectif }\end{array}$ & \# \\
\hline Équivalence & $\begin{array}{l}\text { Lorsque deux éléments sont explicitement donnés pour } \\
\text { équivalents, par exemple par la formule bo kai : « qui (est) } \\
\text { aussi » }\end{array}$ & $=$ \\
\hline
\end{tabular}

- la présence éventuelle de sous-ensembles au sein de la séquence; en l'espèce, deux cas de figure sont possibles :

- deux éléments (ou plus) forment un syntagme; ils sont alors regroupés dans des crochets droits : [élément 1 \# élément 2];

- deux éléments entretiennent le même lien avec un troisième élément, typiquement lorsque deux adjectifs coordonnés qualifient un même substantif; on retranscrit cette construction distributive au moyen de parenthèses: élément 1 \# (élément 2 + élément 3).

Ainsi, dans la dédicace de Timothéa à Artémis Diktunna, si l'on considère que le second élément qualifie le premier, on peut formaliser l'attestation 1. Artemis \# 2. Diktunna; mais compte tenu du fait que Diktunna peut être envisagée comme une puissance autonome ici juxtaposée à Artémis, on retranscrira plutôt 1. Artemis/2. Diktunna. Dans ce type de configuration, l'ordre des éléments n'est pas sans incidence, comme l'a bien montré V. Pirenne-Delforge à propos d'une

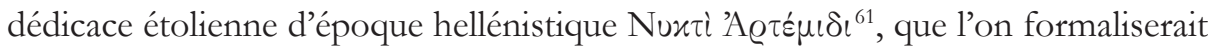
alors 1.Nux/2. Artemis.

Ces instruments de formalisation permettent de prendre en compte les séquences onomastiques dans toute leur variété et complexité, ce que ne permettait pas la $B D E G$, compte tenu de la rigidité de sa structure, construite sur le modèle théonyme + épithète. Si l'on considère par exemple la dédicace suivante ${ }^{62}$ :

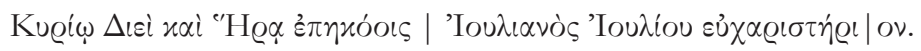

61. Pirenne-Delforge (2018) à propos de SEG 56, 601 (Kallipolis, IV ${ }^{\mathrm{e}-I I I}{ }^{\mathrm{e}} \mathrm{s}$.).

62. SEG 25, 727 = IGBulg V 5286 (Padarino, entre Marcianopolis et Nicopolis de l'Istros. II ${ }^{e}$ s. de n. è.). 
À Kurios Zeus et Héra Epēkooi,

Ioulianos (fils) de Ioulios (a dédié ce relief) en signe de gratitude.

L'épithète Kurios, ici au singulier, se rapporte à Zeus seul, alors qu'Epêkoos, au pluriel, qualifie et Zeus (en l'occurrence Kurios Zeus) et Héra. Par conséquent, cette séquence ne peut être exactement retranscrite dans la $B D E G^{63}$. En revanche, la BDD MAP rend possible une formalisation qui conserve l'unité de l'attestation, ouvrant la voie à un traitement quantitatif des occurrences de chaque élément, tout en retranscrivant la position et l'agencement exact de ces derniers. Pour le dire autrement, la retranscription dans la BDD MAP par la formule suivante : ([1. Kurios \# 2. Zeus] + 3. Hēra) \# 4. Epēkoos ${ }^{64}$ indique que, dans cette occurrence, c'est Zeus, en tant que Kurios, et Héra qui sont ensemble Epēkooi, ce qui ne dénote, a priori, pas exactement la même chose que si Zeus et Héra avaient été chacun, mais de façon séparée, Epēkoos. Selon la même logique, une inscription identifiant un autel comme propriété de Zeus Phratrios et Athéna Phratria ${ }^{65}$ sera formalisée comme suit: [1. Zeus \# 2. Phratrios] + [3. Athêna \# 4. Phratrios], faisant ainsi apparaître la configuration récurrente dans laquelle un binôme Zeus/Athéna est construit sur l'attribution, à chacun, de la même épithète (respectivement déclinée au masculin et au féminin) ${ }^{66}$.

La formalisation rend compte de toutes les nuances inhérentes à l'agencement des séquences onomastiques qui, même proches, ne sont pas identiques. Ainsi ces deux titulatures de prêtres attestées à Kédrées (Pérée rhodienne) aux $\mathrm{II}^{\mathrm{e}}-\mathrm{I}^{\mathrm{er}}$ siècles :

\begin{tabular}{|c|c|}
\hline I.Rhod.Per. 553 (ca. 200-175), 1.9 & I.Rhod.Per. 557 (ca. 150-50), 1. 1-3 \\
\hline 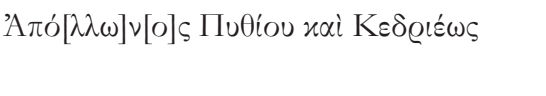 & 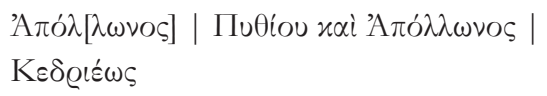 \\
\hline 1. Apollōn \# (2. Puthios +3 . Kedrieus) & $\begin{array}{l}\text { [1. Apollōn \# 2. Puthios }]+[3 \text {. Apollōn } \\
\text { \# 4. Kedrieus }]\end{array}$ \\
\hline
\end{tabular}

63. En l'occurrence la solution retenue (la moins mauvaise) a été de créer deux fiches, une enregistrant Zeus et Héra dans le champ «Nom de la divinité » et Epēkooi dans le champ "Épiclèse », et une autre avec Zeus dans le champ « Nom de la divinité » et Kurios dans le champ «Épiclèse ». L'inconvénient étant que l'on perd trace de la cooccurrence de Kurios et d'Epēkoos. C'est également une illustration, parmi d'autres, que si la BDEG reste très utile en tant que répertoire, elle ne permet aucun traitement quantitatif fiable, et donc exploitable, des données, puisqu'elle altère le rapport entre l'occurrence documentaire et son enregistrement.

64. Dans la formule, les éléments sont indiqués dans leur état absolu, c'est-à-dire, en grec, au nominatif masculin singulier. Le genre, le cas et le nombre sont toutefois enregistrés tels qu'ils apparaissent dans chaque attestation.

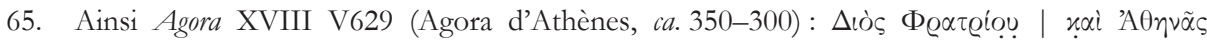

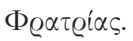

66. Cf. Paul (2010) à partir de la documentation de Cos, mais la configuration se rencontre très fréquemment tout autour du bassin égéen. 
La légère différence de formulation peut certes n'être que le fruit du souci du lapicide de faire plus ou moins court; mais, si on la prend à la lettre, elle peut aussi théoriquement être révélatrice d'évolutions du sacerdoce d'Apollon Pythien et Kédrien à Kédrées, potentiellement unique dans un premier temps, puis scindé en deux prêtrises pouvant être de fait cumulées par un même individu (ce que la documentation locale, faute de compléments, ne permet pas d'étayer). La mise en formule sert donc à aiguiser le regard et les questionnements.

Dans une «attestation » ou « séquence », on voit en effet souvent associées plusieurs facettes d'une même puissance divine ou plusieurs puissances réunies dans une configuration plurielle, ou encore des appellations données pour équivalentes. On combine ces caractéristiques dans cette inscription ptolémaïque de Satis (Haute-Égypte) ${ }^{67}$ :

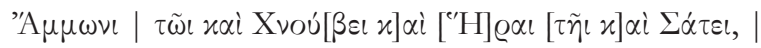

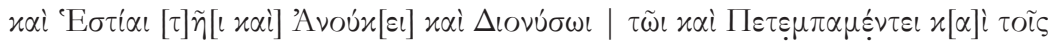

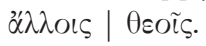

que l'on retranscrira comme suit

$$
\begin{aligned}
& \text { [1. Ammōn }=2 . \text { Chnoubis }]+[3 . \text { Hēra }=4 . \text { Satis }] \\
& +[5 . \text { Hestia }=6 . \text { Anoukis }]+[7 . \text { Dionusos }=8 . \text { Petempamentis }] \\
& +[9 . \text { Hallos \# 10. Theos }]
\end{aligned}
$$

Une telle mise en formule affine notre perception des processus d'interpretatio qui constituent évidemment un terrain d'enquête du projet MAP. De même, dans la perspective d'une approche comparative à l'échelle de la Méditerranée, les formules ont été pensées pour retranscrire aussi bien les attestations tirées de la documentation grecque que sémitique, comme celle que l'on trouve de façon récurrente sur les stèles du tophet de Carthage d'époque punique (e.g. CIS I 184, 1. 1-2) :

\section{lrbt ltnt pn b'l w l'dn lb'l hamn}

À la Dame à Tanit Face de Baal et au Seigneur à Baal Hamon.

Formalisée de la façon suivante

$$
\left[r b t \#\left[t n t \#\left[p n \# b^{c} l\right]\right]\right]+\left[{ }^{\prime} d n \#[b l \# b m n]\right]
$$

La prise en compte de l'ordre des éléments, et des relations entre eux au sein des chaînes, permettra notamment de prendre la mesure de l'importance (numérique), de l'évolution dans le temps et dans l'espace de la «structure » théonyme + épithète $^{68}$.

67. IThSy 302 (c. 152-145), 1. 3-7.

68. Il sera ainsi possible de tester l'assertion de PARKER (2017a) relative à l'allongement des séquences onomastiques divines à partir de l'époque hellénistique et de discuter son lien supposé avec une influence orientale. 


\section{Conclusion}

Au terme de cette présentation, il faut rappeler qu'une base de données n'est pas une finalité en soi, mais un outil indispensable pour réaliser les analyses quantitatives et qualitatives portant sur un corpus aussi étendu que celui des noms attribués aux dieux dans les mondes grec et sémitique. On attirera aussi l'attention sur le fait que la BDD MAP présente un biais important: les attestations des "théonymes» seuls ne sont pas prises en compte. En termes de faisabilité, un projet de cinq ans doit nécessairement calibrer ses objectifs; la priorité a donc été donnée aux appellations composites (à partir de deux éléments) qui seules donnent accès aux façons de qualifier les dieux ou de les combiner. La BDD est évidemment apte à accueillir aussi les attestations d'Aphrodite seule ou de Tanit seule; il faudra, au terme du projet MAP, tenter d'intégrer aussi ces données.

L'outil que nous venons de présenter est destiné à rendre possible des analyses par dieu, par élément, par région, par site, par langue, par période, par agent, par catégorie sémantique, par pratique associée, etc., y compris les innombrables croisements entre ces requêtes. Vous rêvez de savoir comment les marins qualifiaient les dieux sous la protection desquels ils se plaçaient en Grèce insulaire, entre le $\mathrm{VI}^{\mathrm{e}}$ et le $\mathrm{II}^{\mathrm{e}}$ siècle av. n.è.? Et vous souhaitez en outre comparer les résultats obtenus avec ceux qui touchent aux marins travaillant en Sicile et Sardaigne? Ou avec les marins phéniciens et puniques opérant dans les mêmes zones? Vous aimeriez connaitre l'ensemble des divinités auxquelles Apollon est associé dans des séquences onomastiques, les visualiser sur une carte, séquencer les cartes selon des tranches chronologiques, savoir quand il est cité en premier, quand il est juxtaposé ou coordonné à ces autres divinités? Voilà juste quelques pistes possibles. On peut faire le pari que l'outil stimulera des questionnements nouveaux, lesquels en retour feront évoluer l'outil, selon un cercle vertueux destiné à vivifier les recherches portant sur les religions du monde méditerranéen. Qui plus est, la BDD MAP peut être adaptée à d'autres aires géoculturelles, comme le monde latin, mésopotamien, égyptien... Quelques contacts ont déjà été pris dans ce sens.

Pour exploiter la BDD au mieux, nous sommes occupés à concevoir des outils qui permettront de traiter les données enregistrées par le biais d'analyses statistiques et d'analyses de réseaux (Social Network. Analysis) reposant sur les liens (ou l'absence de liens) que les séquences onomastiques font apparaitre. Deux divinités mentionnées ensemble dans une même séquence sont reliées; deux divinités partageant un même élément onomastique sont reliées. La visualisation des résultats sous forme de graphes, donc de configurations plus ou moins remarquables, sera une fois encore un instrument mis au service des analyses qualitatives. Il ne s'agit pas de « jouer» avec de jolis graphes, mais de les mettre au service de l'interprétation des données. Le recours aux outils de webmapping, c'est-à-dire de cartographie dynamique des données, répondent à la même ambition, l'espace étant, comme on l'a souligné ci-dessus, une composante majeure de la qualification des dieux et de leurs associations. Or rendre visibles les agencements panthéoniques de tel 
ou tel lieu, de telle ou telle époque, autour de tel ou tel dieu permettra assurément de mieux comprendre les logiques qui sous-tendent les systèmes religieux. Ces approches ont pour objectif de dépasser un stade empirique, en grande partie intuitif, et d'atteindre une appréhension structurelle et dynamique des mondes divins.

Une extension possible de la BDD MAP, à laquelle nous réfléchissons, mais que nous pourrons tout au plus tester sur un corpus limité, consiste à explorer les connexions possibles entre les éléments ou attributs onomastiques composant les attestations ou séquences et les attributs iconographiques. Notre premier article collectif se nourrit du reste d'une citation de Julien : «Les dénominations des dieux nous offrent comme autant d'images dessinées ${ }^{69}$ ", qui suggère un dialogue possible entre les noms et les représentations figurées. Certes, la notion d'« attribut iconographique » fait débat chez les historiens de l'art et il n'est en aucun cas question de postuler une relation univoque, ou pire bi-univoque, entre un nom et une image $($ Zeus $=$ foudre $)$. Comme pour les attributs onomastiques, les images construites et mobilisées pour parler des dieux sont complexes, combinatoires, entre tradition et créativité. L'égide d'Athéna, pour prendre un exemple célèbre, est commune à Zeus, parfois aussi à Apollon. Ce partage, comme le partage de la qualification de Polieus — Polias, exclusive du couple Père/Fille, est pourtant hautement significatif et mérite d'être analysé. Avec les spécialistes de la mise en images des dieux, grecs ou sémitiques, un dialogue est certainement souhaitable et du reste déjà engagé. À terme, il pourrait peut-être conduire à une autre extension de la BDD MAP. Pour l'heure, notre priorité est de nourrir cette base de données et de commencer à produite les premiers résultats exploitables. Il reste au projet MAP trois ans pour tendre vers l'exhaustivité.

Sylvain LEBRETON

Corinne BONnET

Université Toulouse - Jean Jaurès, PLH-ERASME

ERC Advanced Grant « Mapping Ancient Polytheisms » (MAP)

sylvain.lebreton@univ-tlse2.fr

corinne.bonnet@univ-tlse2.fr

69. Julien, Lettres, 89b, 291 b. Cf. BonNet et al. (2018). 


\section{Bibliographie}

G. Adorni, M. Maratea, L. Pandolfo, L. Pulina, «An Ontology for Historical Research Documents », in B. TEn Cate, A. Mileo (éd.), Web Reasoning and Rule Systems, Heidelberg, 2015, p. 11-18.

N. Belayche et al. (éd.), Nommer les Dieux. Théonymes, épithètes, épiclèses dans l'Antiquité, Turnhout, 2005.

J.-F. BERT, Une histoire de la fiche érudite, Villeurbanne, 2017.

C. BONNET, «"L'histoire séculière et profane des religions” (F. Cumont) : observations sur l'articulation entre rite et croyance dans l'historiographie des religions de la fin du XIX et de la première moitié du XXe siècle ", in J. SCHEID (éd.), Rites et croyances dans les religions du monde romain, Genève/ Vandoeuvres, 2007 (Entretiens sur l'Antiquité classique, 53), p. 1-37.

C. Bonnet，M. Bianco，T. Galoppin，E. Guillon，A. Laurent，S. Lebreton，F. Porzia, " "Les dénominations des dieux nous offrent comme autant d'images dessinées" (Julien, Lettres 89b, 291 b). Repenser le binôme théonyme-épithète », SMSR 84 (2018), p. 567-591.

L. Bricault, «Zeus Hélios mégas Sarapis », in C. Cannuyer (éd.), La langue dans tous ses états. Michel Malaise in honorem, Bruxelles/Liège/Louvain-la-Neuve/Leuven, 2005 (Acta Orientalia Belgica, 18), p. 243-254.

C.F.H. BRuchmann, Epitheta deorum quae apud poetas graecos leguntur, Leipzig, 1893 (Ausfübrliches Lexikon der griechischen und römischen Mythologie, suppl. 7.1).

P. BRuLÉ, «Le langage des épiclèses dans le polythéisme hellénique (l’exemple de quelques divinités féminines). Quelques pistes de recherches », Kernos 11 (1998), p. 13-34.

P. Brulé, S. Lebreton, « La Banque de données sur les épiclèses divines (BDDE) du Crescam : sa philosophie », Kernos 20 (2007), p. 217-228.

C. CALAME, «Nommer, qualifier et invoquer les divinités grecques : quelles procédures énonciatives? Quelle pragmatique linguistique et poétique? ", à paraitre.

J.B. CARTER, Epitheta deorum quae apud poetas Latinos leguntur, Leipzig 1902 (Ausfübrliches Lexikon der Griechischen und Römischen Mythologie, suppl. 7.2).

E. Cassirer, Philosophie der symbolischen Formen. 2. Das mythische Denken, Berlin, 1925 (tr. fr. Langage et mythe. A propos des noms de dieux, Paris, 1973).

A. Chaniotis, «Epiklese », Der Neue Pauly 3, Stuttgart/Weimar, 1997, 1118-1121.

J. DeE, Epitheta deorum apud Homerum, Hildesheim, 1994.

R. Gagné, «Sémantiques de l'épithète divine : origines et compétence », in C. BonNet, G. Pironti (éd.), Les Dieux d'Homère III. Euryopa. Embrasser les épithètes divines chez. Homère et dans sa réception, à paraître.

L. Gernet, A. Boulanger, Le Génie grec dans la religion, Paris, 1932 [1970].

M.H. Hansen, T.H. Nielsen (éd.), An Inventory of Archaic and Classical Poleis, Oxford/New York, 2004.

S. Hornblower, «Lykophron and Epigraphy: The Value and Function of Cult Epithets in the Alexandra », CQ 64 (2014), p. 91-120.

N. Ide, D. Woolner, «Historical Ontologies », in K. Ahmad et al. (éd.), Words and Intelligence II, Dordrecht, 2007, p. 137-152 (Text, Speech and Language Technology, 36).

S. Lebreton, «Zeus Polieus à Athènes. Les Bouphonies et au-delà », Kernos 28 (2015), p. 85-110.

T.N.D. MetTinger, In Search of God: The Meaning and Message of the Everlasting Names, Philadelphie, 1988.

R. Parker, «The Problem of the Greek Cult Epithet », OAth 28 (2003), p. 173-183.

—, Greek Gods Abroad: Names, Natures, and Transformations, Oakland, 2017a. 
—, "Zeus Plus », in C. Bonnet et al. (éd.), Puissances divines à l'épreuve du comparatisme : constructions, variations et réseaux relationnels, Turnhout, 2017b, p. 309-320.

M. PARRY, L'Épithète traditionnelle dans Homère. Essai sur un problème de style homérique, Paris, 1928a.

—, Les Formules et la Métrique d'Homère, Paris, 1928b.

—, The Making of Homeric Verse. The Collected Papers of Milman Parry, Oxford, 1971.

St. PAuL, «À propos d'épiclèses «trans-divines»: le cas de Zeus et d'Athéna à Cos », ARG 12 (2010), p. 65-82.

—, " "Pallas étend ses mains sur notre cité". Réflexion sur le paysage épiclétique autour de l'Athéna "poliade" ", Pallas 100 (2016), p. 119-138.

V. Pirenne-Delforge, Retour à la source. Pausanias et la religion grecque, Liège, 2008 (Kernos, suppl. 20).

—, « Nyx est, elle aussi, une divinité : La nuit dans les cultes et les mythes grecs », in A. CHANiótis (éd.), La nuit : Imaginaire et réalités nocturnes dans le monde gréco-romain, Vandoeuvres, 2018 (Entretiens sur l'antiquité classique, 64), p. 131-165.

V. Pirenne-Delforge, J. Scheid, «Vernant, les dieux et les rites : héritages et controverses », in C. Bonnet et al. (éd.), Puissances divines à l'épreuve du comparatisme. Constructions, variations et réseaux relationnels, Turnhout, 2017, p. 33-52.

L. Preller, C. Robert, Griechische Mythologie, vol. 1, Berlin, 1894 (éd. or. 1854).

J. Scheid, J. Svenkro, «Les Götternamen de Hermann Usener : une grande théogonie », in Belayche (2005), p. 93-103.

M.S. SмITH, Where the Gods Are. Spatial Dimensions of Anthropomorphism in the Biblical World, New Haven, 2016.

D. Summa, «L'archivio delle Inscriptiones Graecae (Berlino) », Anabases 16 (2012), p. 267-274.

H. Usener, Götternamen : Versuch einer Lehre von der reliogiösen Begriffsbildung, Bonn, 1896.

J.-P. Vernant, Mythe et pensée chę les Grecs. Études de psychologie historique, Paris, 1965.

—, « La société des dieux », in ID., Mythe et société en Grèce ancienne, Paris 1974, p. 103-120.

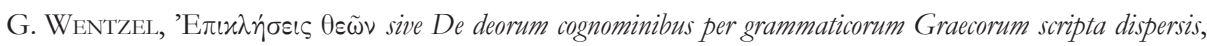
Göttingen, 1889.

\section{CRÉditS PHOTOGRAPHIQUES}

Fig. 1-2 Photographies Sylvain Lebreton, avec l'aimable autorisation de l'Akademie der Wissenschaften de Berlin. 